\title{
REVISIÓN DEL MÉTODO DE PRONÓSTICO DEL TEXTO.
}

ELEMENTOS DE PRODUCCIÓN

Miller Alarcón

\section{RESUMEN:}

Este documento hace una revisión de un método de pronóstico, que se usa en diferentes programas de Ingeniería Industrial y en algunos programas de postgrado de nuestro país, en asignaturas propias del área de producción. El método "Torres", como lo llamaremos de aquí en adelante, se basa en el uso de la técnica de suavización exponencial, basado en el texto de Spyros Makridakis: Método de pronóstico basado en la técnica de suavización exponencial,(México: Limusa, 1997) En el documento se puede observar más a fondo el análisis que se hizo de cada uno de los componentes del método de Torres, y se trata de entender mejor la metodología propuesta, hallar las bondades y debilidades de la misma, y ante todo, mostrar una metodología para determinar uno de los componentes que es dejado a la subjetividad del usuario del método para que se halle de manera objetiva.

Se espera- que esta revisión sirva para que se aproveche mejor este método de pronóstico, que se entiendan sus bondades y también sus limitaciones, y que se añada la manera de hallar el componente subjetivo de una manera más clara. Otro resultado esperado es que, de algún modo, este documento sea un punto de partida para futuras revisiones más profundas, hechas por docentes a otras herramientas que son usadas día a día en las asignaturas y cátedras, y, por supuesto, en esta misma área (los métodos de pronósticos).

\section{ABSTRACT:}

This document reviews a forecasting method used in various industrial engineering programs and in some post-graduate programs in our country, in subjects on production. The "Torres" method, as we will call it here, is based on the Makridakis Spyros text: Forecasting method based on the exponential softening technique (Metodo de pronóstico basado en la técnica de suavización exponencial, México: Limusa, 1997), The analysis made on each one of the components of the Torres method can be observed more in depth in the document. The idea is to better understand the methodology proposed, find its benefits and weaknesses, and above all, show a methodology for determining one of the components which is left to the subjectivity of the user so that it be somehow found objectively.

It is expected that this review will help to better take advantage of this forecasting method, that its benefits and also its limitations be understood, and that a way for finding a subjective component more clearly be added; a further expected result is that this document be in some way a starting point for future, deeper reviews, made by teachers to other tools which are used on a daily basis in subjects and courses, and of course, in this same area (the forecasting methods).

\section{PALABRAS CLAVE:}

Pronóstico, series de tiempo, suavización exponencial, Método Delphi, tendencia, ciclicidad, estacionalidad, Método de Holt, Método de Winters, Coeficiente de suavización, MSE (Minimo error al cuadrado).

\section{KEYWORDS:}

Forecast; time series, exponential smoothing, Delphi method, trend, cyclicity, seasonal variation, Holt's Method, Winters' Method, smoothing coefficient, minimum square error (MSE) 


\section{INTRODUCCIÓN}

Los pronósticos son una de las herramientas con las que el Ingeniero Industrial se prepara frente al futuro, para planear de una mejor manera los posibles comportamientos de las variables, tomar decisiones que le permitan ser eficiente, y no se verse sorprendido por situaciones previsibles. Pronóstico es un procedimiento objetivo, "en el que se utiliza información recabada en un espacio de tiempo, para extrapolarlo en el tiempo"1. Se encontró que el método analizado, usa la subjetividad para determinar uno de los datos de entrada del modelo de modo que se ajuste más a las condiciones de la serie; esta particularidad no se halla en ningún otro método, $y$ fue por ello que llamó la atención, y motivó ésta revisión. Por otro lado, una razón adicional para examinar en detalle esta herramienta es que algunos métodos y herramientas que se usan en muchas de las asignaturas que se imparten a nuestros estudiantes, se dan por ciertos e indiscutibles y no son sujetos de revisión, actualización o refutación.

\section{RESEÑA DEAUTOR}

Miller Alarcón. Es Ingeniero Industrial y especialista en Ingeniería de Producción de la Universidad Distrital Francisco José de Caldas. Actualmente se desempeña como docente de la facultad de Ingeniería Industrial del Politécnico Grancolombiano y es catedrático de pregrado y postgrado en las universidades Rosario y Externado de Colombia.
Se quiere con el método de Torres ${ }^{2}$, describir su fundamentación (basado en la técnica de suavización exponencial ${ }^{3}$ ), su utilización (a partir del texto ${ }^{4}$ del cual se extrajo), tratar de encontrar las ventajas y desventajas del mismo y aclarar su pertinencia dentro de las asignaturas que tienen que ver con planeación de la producción en los programas de la Ingeniería Industrial.

\section{DESCRIPCIÓN DELOS PRONÓSTICOS}

Los métodos de pronósticos pueden ser de dos grandes grupos: los que están basados en técnicas de demanda derivada y los métodos que están basados en series de tiempo. Los primeros se enfocan en variables de demanda de productos; son nombrados así por la forma como se trabajan los datos de la demanda para inferir el comportamiento de la misma; son muy sencillos y se fortalecen con el análisis de la variable y el conocimiento que se tenga del mercado y de las variables externas. Los segundos son más estructurados y permiten predecir el comportamiento de una variable en el futuro, de acuerdo con métodos o modelos que tienen en cuenta la tendencia, el ciclo, la estacionalidad y el azar.

En el primer grupo se encuentran los métodos de oscilación ${ }^{5}$, en los cuales se

1. Definición tomada de la APICS (American Production and Inventory Control Society), FOGARTY, Donald. Administración de la producción e inventarios, 1999. Editorial CECSA. Pág. 99 Falta dato sobre la ciudad de la edición del libro

2. En el presente documento se llamará método de Torres al método analizado, de acuerdo con su autor, TORRES ACOSTA, Jairo Humberto. Elementos de producción. Editorial Universidad Católica.

3. El método de suavización exponencial se describe ampliamente en MAKRIDAKIS, Spyros (1997). Métodos de Pronóstico.. México: Editorial Limusa. Página 79, Faltan datos sobre la ciudad donde fue editado el libro- Las diferencias del método de Torres frente al método de suavización exponencial se tratarán detalladamente a lo largo de este documento.

4. El texto en el que se encuentra descrito el método que se analiza en este documento es Elementos de producción, Bogotá: Universidad Católica de Colombia, Facultad de Ingeniería Industrial. s.f. De acuerdo con el uso de este método en la Universidad Distrital se intuye que fue publicado entre los años 1995 y 2000.

5. Este método es descrito con mayor detalle en TORRES ACOSTA, Jairo, Op. Cit.. Pág. 55. 
desarrollan análisis previos de la tendencia y de los factores macroeconómicos que afectan la demanda de un producto, para a través de esta inferencia, tratar de anticipar los cambios en el comportamiento de la variable analizada.

Otra de las técnicas de oscilación es el método de indicadores acopiados o agrupados, en el que se analiza el comportamiento de un producto para predecir el comportamiento de otro, porque se pueden establecer relaciones entre uno y otro. Para este caso, la técnica puede tratar con productos sustitutos o complementarios, o porque el mercado y el producto son muy similares y se espera que se comporten de la misma manera.

En el segundo grupo, las técnicas son las relacionadas con las series de tiempo ${ }^{6}$, las series de tiempo tienen cuatro componentes fundamentales o factores subyacentes ${ }^{7}$ : la tendencia, el ciclo, la estacionalidad y el error, (azar o la aleatoriedad), para este último, lo que existen son técnicas que puedan minimizarlo; un ejemplo son las técnicas de medir el error mínimo al cuadrado, para saber cuál tiene menor dispersión con respecto a los datos reales.

Lo que ningún método de pronóstico hace es contemplar el error o azar dentro de un modelo matemático que represente las relaciones de las variables de una demanda; pero para los tres primeros componentes sí, y es gracias a estos tres componentes como se puede crear un modelo que permita tratarlos de alguna manera.

En la siguiente tabla se puede ver una clasificación de los diferentes métodos de pronóstico, así como su calificación en cuanto al uso, de acuerdo con el horizonte de tiempo para el cual fueron diseñados y el costo asociado para cada método:

Tabla 1. Clasificación de los diferentes métodos de pronóstico ${ }^{8}$

\begin{tabular}{|l|l|l|l|}
\hline \multicolumn{1}{|c|}{ MÉTODO } & \multicolumn{1}{|c|}{ BREVE DESCRIPCIÓN } & HORIZONTE & COSTO \\
\hline Análisis de ventas & Estimación del área de ventas como un todo & CP-MP & B-M \\
\hline Opinión ejecutiva & $\begin{array}{l}\text { Gerentes de mercadotecnia, finanzas y producción preparan } \\
\text { pronósticos }\end{array}$ & CP-LP & B-M \\
\hline Ventas y Gerentes & $\begin{array}{l}\text { Los cálculos independientes de los vendedores regionales son } \\
\text { canalizados con proyecciones nacionales de los gerentes de línea } \\
\text { de productos }\end{array}$ & MP & M \\
\hline Analogía histórica & $\begin{array}{l}\text { Pronóstico proveniente de la comparación con un producto similar } \\
\text { previamente introducido }\end{array}$ & CP-LP & B-M \\
\hline Delphi & $\begin{array}{l}\text { Los expertos responden (anónimamente) una serie de preguntas, } \\
\text { reciben retroalimentación y revisan sus cálculos. }\end{array}$ & LP & M-A \\
\hline $\begin{array}{l}\text { Investigaciones de } \\
\text { mercado }\end{array}$ & $\begin{array}{l}\text { Se usan cuestionarios y paneles para obtener datos que anticipen } \\
\text { el comportamiento del consumidor. }\end{array}$ & MP-LP & A \\
\hline
\end{tabular}

6. MAKRIDAKIS, Spyros. Op.Cit. Pág. 75

7. FOGARTY, Donald (1999). Administración de la producción e inventarios, Editorial CECSA. Pág. 102 Faltan datos.

8. Aunque en esta clasificación no aparece el método de Torres, se debe clasificar en la categoría de series de tiempo, y en la subcategoría de los de suavización exponencial, debido a que este usa el método de suavización exponencial para calcular los pronósticos. 


\begin{tabular}{|l|l|l|l|}
\hline \multicolumn{1}{|c|}{ MÉTODO } & \multicolumn{1}{|c|}{ BREVE DESCRIPCIÓN } & HORIZONTE & COSTO \\
\hline \multicolumn{3}{|c|}{ SERIE DE TIEMPOS (CUANTITATIVOS) } & B \\
\hline Promedio simple & $\begin{array}{l}\text { Se usa una regla simple que pronostica igual al promedio de los } \\
\text { datos históricos. }\end{array}$ & CP & B \\
\hline Promedios móviles & $\begin{array}{l}\text { El pronóstico es simplemente un promedio de los n más } \\
\text { recientes. }\end{array}$ & CP & B \\
\hline $\begin{array}{l}\text { Proyección de la } \\
\text { tendencia }\end{array}$ & $\begin{array}{l}\text { El pronóstico es una proyección lineal, exponencial u otra de la } \\
\text { tendencia pasada. }\end{array}$ & MP-LP & B \\
\hline $\begin{array}{l}\text { Descomposición } \\
\text { Holts-Winters }\end{array}$ & $\begin{array}{l}\text { Las series de tiempos se dividen en sus componentes de } \\
\text { tendencia: estacional cíclica y aleatoria, }\end{array}$ & CP-LP & B \\
\hline $\begin{array}{l}\text { Suavización } \\
\text { exponencial }\end{array}$ & $\begin{array}{l}\text { Los pronósticos son promedios móviles ponderados } \\
\text { exponencialmente, donde los últimos valores tienen mayor peso. }\end{array}$ & CP & M-A \\
\hline Box-jenkins & $\begin{array}{l}\text { Se propone un modelo de regresión de serie de tiempo, } \\
\text { estadísticamente probado modificado y vuelto a probar hasta que } \\
\text { sea satisfactorio. }\end{array}$ & MP-LP & ASoclATIVos (CUANTITATIVos O CAUSALES) \\
\hline Regresión y correlación & $\begin{array}{l}\text { Se usan una o más variables asociadas para pronosticar por } \\
\text { medio de la ecuación de mínimos cuadrados (regresión) o de una } \\
\text { asociación (correlación) con una variable explicativa. }\end{array}$ & CP-MP & M-A \\
\hline Econométricos & $\begin{array}{l}\text { Se usa una solución por ecuaciones simultáneas de regresión } \\
\text { múltiple para una actividad económica }\end{array}$ & CP-LP & A \\
\hline
\end{tabular}

Abreviaturas: $\mathrm{B}=$ bajo, $\mathrm{M}=$ medio, $\mathrm{A}=$ alto, $\mathrm{CP}=$ corto plazo, $\mathrm{MP}=$ mediano plazo, $\mathrm{LP}=$ largo plazo.

Tomado de: PAREDES, Jorge (2001). Planificación de la producción, Universidad de Cuenca.

Algunos métodos no tienen en cuenta los tres elementos mencionados, o le dan más peso a alguno de ellos. Por ejemplo, el método de Holt-Winter's ${ }^{9}$ es un método que le da mayor importancia a la tendencia de los datos sobre los demás componentes de las series de tiempo.

Para aclarar más las cosas, se puede mencionar que "la tendencia, se refiere al comportamiento general de una variable dentro de un período definido, y son las líneas rectas que van aumentando o disminuyendo de manera proporcional en cada uno de los períodos analizados" ${ }^{10}$. Es una línea que se ajusta al patrón histórico en el horizonte de tiempo. Se puede decir que la tendencia es la regresión lineal de los datos, y usualmente se calcula con el promedio móvil para eliminar las variaciones tales como la estacionalidad. El ciclo es un comportamiento de largo plazo, y por ende, requiere mucha información histórica para analizar los comportamientos que se repiten a través de la serie de tiempo. La estacionalidad se refiere a patrones del comportamiento de la variable en los mismos períodos de tiempo; por ejemplo, las temporadas de venta de algunos artículos

9. El método de Holt se explica a profundidad en MAKRIDAKIS, Spyros. Op.Cit. Pág. 87.

10. Definición tomada de ACOSTA TORRES, Jairo Humberto. Op.Cit. Pág. 51. 
marcan los períodos de mayores y menores ventas del producto y este patrón de comportamiento se repite año tras año. La idea es poder separar los comportamientos de estacionalidad, y de tendencia y ciclo para modelarlos adecuadamente.

\section{DESCRIPCIÓN DE LOS PRONÓSTICOS EN EL MÉTODO DE TORRES}

El método que analizaremos se fundamenta en el método de suavizado exponencial, y al igual que el método de Holt, trabaja con el método de suavizado doble o corregido, el fundamento es el mismo: el cálculo correspondiente requiere de dos datos: el primero es la demanda real del período más reciente y el segundo es el pronóstico más reciente obtenido por cualquier otro método.

A continuación se muestra la fórmula del método de Suavizado Exponencial que es la base de ambos métodos, el de Holt y el de Torres:

Pronóstico de la demanda del período $t+1=\alpha\left(\begin{array}{l}\text { Demanda } \\ \text { más reciente }\end{array}\right)+(1-\alpha) \times\left(\begin{array}{l}\text { Pronóstico } \\ \text { más reciente }\end{array}\right)$

esdecir; $F t=\left(\alpha \times D_{t-1}\right)+(1-\alpha) \times S_{t-1}$

Donde:

$\mathrm{Ft}=$ Pronóstico del período $\mathrm{t}$

$\alpha=$ Coeficiente de suavización

$\mathrm{S}_{\mathrm{t}-1}=$ Dato ajustado o calculado del período ${ }_{\mathrm{t}-1}$

$\mathrm{D}_{\mathrm{t}-1}=$ Demanda tomada como base para

el pronóstico ${ }^{11}$

Se llama exponencial este método porque, si se lo aplica para varios períodos en forma sucesiva, se puede obtener una fórmula como la siguiente:

$\mathrm{Ft}=\left[\alpha(1-\alpha)^{0} D_{t-1}\right]+\left[\alpha(1-\alpha)^{1} D_{t-2}\right]+\left[\alpha(1-\alpha)^{2} D_{t-3}\right] \ldots \ldots \ldots+(1-\alpha)^{3} \times F_{t-3}$

Los coeficientes $\alpha$ pueden variar entre 0 y 1 , tomando cualquier valor. El peso de los coeficientes determina la confiabilidad del método, si el coeficiente de suavización $\alpha$ es cercano a cero, tendrán mayor peso los valores más recientes en la serie de datos históricos, pero si el coeficiente de suavización $\alpha$ es cercano a uno, entonces se les dará más peso a los datos más lejanos en la serie de tiempo.

11. Más adelante se verificará que para Dt-1 (la demanda tomada como base histórica) no se deben tomar grandes colecciones de datos; debido a la naturaleza del método, es mejor tomar las demandas más recientes. 
Un elevado coeficiente de suavización sería más adecuado para los nuevos productos o para casos para los que la demanda subyacente está en proceso de cambio (ésta es dinámica, o bien inestable). Un valor del coeficiente de suavización $\alpha$ del rango 0.7 a 0.9 puede resultar el más apropiado para estas condiciones, aun cuando el uso del suavizado exponencial es cuestionable si no se sabe si existen o no condiciones de inestabilidad. Si la demanda es muy estable y se piensa que pueda ser representativa del futuro, el pronosticador podrá optar por un valor bajo de coeficiente de suavización $\alpha$ para disminuir cualquier ruido que hubiera podido presentarse en forma súbita; entonces, el procedimiento de pronóstico no reacciona de una manera drástica a las demandas más recientes. En estas condiciones de estabilidad, el coeficiente de suavización podría estar en un rango entre 0.1 a 0.3 . Cuando la demanda es ligeramente inestable, se recomiendan coeficiente de suavización $\alpha$ en un rango entre 0.4 , a un rango 0.6 , puede proporcionar los pronósticos más precisos. ${ }^{12}$

El método de Torres, contempla la utilización de los coeficientes de suavización $\alpha$, desde 0.1 hasta 0.9 . Esta es la primera diferencia que encontramos con el método de suavización exponencial tradicional ${ }^{13}$, que recomienda elegir un solo coeficiente de suavización $\alpha$ para realizar los cálculos que producirán el pronóstico.

El método de Torres, por el contrario trabaja con 9 coeficientes de suavización $\alpha, 0.1,0.2,0.3,0.4,0.5,0.6,0.7,0.8$ y $0.9, y$ no menciona la posibilidad de trabajar con valores intermedios entre ellos.
El método de Torres presenta la siguiente metodología:

1. Cálculo del pronóstico simple

$$
\mathrm{S}_{\mathrm{t}+1}=\alpha \times X_{t}+(1-\alpha) \times \mathrm{S}_{\mathrm{t}}
$$

Donde, $\mathrm{X}_{\mathrm{t}}$ se refiere a la demanda histórica en cada período de tiempo

2. Cálculo del pronóstico doble

$$
\mathrm{S}_{\mathrm{t}+1}=\alpha \times S_{t+1}+(1-\alpha) \times \mathrm{S}_{\mathrm{t}}
$$

3. Cálculo del pronóstico de ajuste

$$
\begin{aligned}
& S_{t+1}^{\wedge}=a+b \\
& a=\left(2 \times S_{t+1}\right)-S_{t+1}^{i} \\
& \mathrm{~b}=\frac{\alpha}{1-\alpha} \times\left(\mathrm{S}_{\mathrm{t}+1}-\mathrm{S}_{\mathrm{t}+1}^{\odot}\right)
\end{aligned}
$$

Para los pronósticos simple y doble, Torres los ajusta siempre por exceso, es decir, no contempla la posibilidad de trabajar con fracciones decimales, no hace aproximación aritmética, siempre los aproxima al entero siguiente.

El método de Torres, presenta además, otras diferencias; una es la incorporación del pronóstico de ajuste, usando para ello los coeficientes a y b; otra es que el método de suavizado exponencial sirve para pronosticar sólo un dato a partir de la serie de datos históricos, el de Torres parece ser bondadoso en ese sentido y permite pronosticar el mismo número de datos de entrada, y la última diferencia encontrada en este apartado es que no pierde el primer dato calculado como sí lo hace el método tradicional ${ }^{14}$. Para ello calcula 
el primer dato ajustado como el promedio de los demás datos ajustados; es decir, que para calcular el primer dato, primero se deben calcular los restantes y al final promediarlos.

En la hoja de cálculo se puede formular con facilidad este aspecto desde el inicio, y se ajustará a medida que la formula de los ajustados se replique.
Para aclarar el uso del método de Torres, hasta aquí descrito, a continuación se muestra un pequeño ejemplo de los cálculos mencionados usando para ello una serie de cinco datos históricos. Cabe mencionar que el coeficiente de suavización $\alpha$ usado para este ejemplo es de 0.3

Tabla 2. Veamos como se realizaron los cálculos:

\begin{tabular}{|c|c|c|c|c|c|c|c|c|c|c|}
\hline \multirow{2}{*}{ Período } & \multicolumn{3}{|c|}{\begin{tabular}{c} 
PRONÓSTICO SIMPLE \\
\cline { 2 - 12 }
\end{tabular}} & \multicolumn{2}{c|}{$\begin{array}{c}\text { PRONÓSTICO } \\
\text { DOBLE }\end{array}$} & \multicolumn{2}{c|}{ VALOR DE a } & \multicolumn{2}{c|}{ VALOR DE b } & PRON \\
\cline { 2 - 12 } & DEM $^{15}$ & CÁLCUL016 & AJUSTE & CÁLCULO & AJUSTE & CÁLCULO & AJUSTE & CÁLCULO & AJUSTE & AJUSTE \\
\hline $\mathbf{1}$ & $\mathbf{5 0 0}$ & 628,85 & 629 & 634,11 & 635 & 623,59 & 624 & $-2,25$ & -2 & 622 \\
\hline $\mathbf{2}$ & $\mathbf{7 0 0}$ & 500,00 & 500 & 628,85 & 629 & 371,15 & 372 & $-55,22$ & -55 & 317 \\
\hline $\mathbf{3}$ & $\mathbf{9 0 0}$ & 560,00 & 560 & 608,20 & 609 & 511,81 & 512 & $-20,66$ & -20 & 492 \\
\hline $\mathbf{4}$ & $\mathbf{1 1 0 0}$ & 662,00 & 662 & 624,34 & 625 & 699,66 & 700 & 16,14 & 17 & 717 \\
\hline $\mathbf{5}$ & $\mathbf{1 3 0 0}$ & 793,40 & 794 & 675,06 & 676 & 911,74 & 912 & 50,72 & 51 & 963 \\
\hline
\end{tabular}

\section{PRONÓSTICO SIMPLE}

Para el primer período el 628.85 es el promedio de los cálculos de los períodos 2 , 3,4 y 5 , es decir promedio $(500,700,560$, 662, 793.40).

Para el segundo período, el 500 hace referencia al primer dato histórico.

Del tercer período en adelante se hace uso de la fórmula para el pronóstico simple mencionada por Torres:

$$
\begin{aligned}
& \mathrm{S}_{\mathrm{t}+1}=\alpha \times X_{t}+(1-\alpha) \times \mathrm{S}_{\mathrm{t}} \\
& 560=(0.3 \times 700)+(0.7 \times 500) \\
& 662=(0.3 \times 900)+(0.7 \times 560) \\
& 793.40=(0.3 \times 1100)+(0.7 \times 662)
\end{aligned}
$$

Los ajustes se hacen aproximando por encima los cálculos del pronóstico simple.

\section{PRONÓSTICO DOBLE}

El 634.11 es el promedio de los cálculos de los períodos 2, 3, 4 y 5; es decir, promedio $(628.85,608.20,324.34,675.06)$.

Para el segundo período, el 628.85 hace referencia al primer dato calculado del pronóstico simple

Del tercer período en adelante se hace uso de la fórmula para el pronóstico doble mencionada por Torres:

$$
\begin{aligned}
& \mathrm{S}_{\mathrm{t}+1}=\alpha \times S_{t+1}+(1-\alpha) \times \mathrm{S}_{\mathrm{t}} \\
& 608.20=(0.3 \times 560)+(0.7 \times 628.85) \\
& 624.34=(0.3 \times 662)+(0.7 \times 608.20) \\
& 675.06=(0.3 \times 793.40)+(0.7 \times 624.34)
\end{aligned}
$$

Los ajustes se hacen aproximando por encima los cálculos del pronóstico simple.

14. MAKRIDAKIS, Spyros. Op. Cit. Pág. 77.

15. DEM, se refiere a la demanda histórica tomada como base para hacer el pronóstico

16. Estos cálculos fueron hechos con un coeficiente de suavización $\alpha$ de 0.3 , la idea es replicar estos cálculos para todos los coeficientes de suavización $\alpha$, que se vayan a utilizar 


\section{VALOR DE $\alpha$}

El 623.59 es el promedio de los cálculos de los períodos 2, 3, 4 y 5; es decir, promedio $(371.15,511.81,699.66,911.74)$.

Del segundo período en adelante se hace uso de la fórmula para el valor de a mencionada por Torres:

$$
\begin{aligned}
& a=\left(2 \times S_{t+1}\right)-S_{t+1}^{i} \\
& 371.15=(2 \times 500)-628.85 \\
& 511.81=(2 \times 560)-608.20 \\
& 699.66=(2 \times 662)-624.34 \\
& 911.74=(2 \times 793.40)-675.06
\end{aligned}
$$

Los ajustes se hacen aproximando por encima los cálculos del pronóstico simple.

\section{VALOR DEb}

El -2.25 es el promedio de los cálculos de los períodos $2,3,4$ y 5 , es decir, el promedio (-55.22, -20.66, 16.14, 50.72).

Del segundo período en adelante se hace uso de la fórmula para el valor de b mencionada por Torres:

$$
b=\frac{\alpha}{1-\alpha} \times\left(S_{t+1}-S_{t+1}^{\prime}\right)
$$

$$
\begin{aligned}
& -55.22=(0.3 / 0.7) \times(500-628.85) \\
& -20.66=(0.3 / 0.7) \times(560-608.20) \\
& 16.14=(0.3 / 0.7) \times(662-624.34) \\
& 50.72=(0.3 / 0.7) \times(793.4-675.06)
\end{aligned}
$$

Los ajustes se hacen aproximando por encima los cálculos del pronóstico simple.

\section{PRONÓSTICO}

Para todos los cinco períodos se usa la siguiente fórmula, (nótese que los valores de a y b son los ajustados):

$$
\begin{aligned}
& S_{t+1}^{\wedge}=a+b \\
& 622=624+(-2) \\
& 317=372+(-55) \\
& 492=512+(-20) \\
& 717=700+17 \\
& 963=912+51
\end{aligned}
$$

A continuación se muestran los cálculos usando otros dos coeficientes de suavización $\alpha$, en este caso 0.5 y 0.7 , además del presentado anteriormente, 0.3 :

Tabla 3. Cálculos para coeficiente de suavización $\alpha=0.3$

\begin{tabular}{|c|c|c|c|c|c|c|c|c|c|}
\hline \multirow{2}{*}{ Período } & \multicolumn{2}{|l|}{ PRONÓStICO SIMPLE } & \multicolumn{2}{l|}{ PRONÓSTICO DOBLE } & \multicolumn{2}{c|}{ VALOR DE a } & \multicolumn{2}{c|}{ VALOR DE b } & PRON \\
\cline { 2 - 11 } & CÁLCULO & AJUSTE & CÁLCULO & AJUSTE & CÁLCULO & AJUSTE & CÁLCULO & AJUSTE & AJUSTE \\
\hline 1 & 628,85 & 629 & 634,11 & 635 & 623,59 & 624 & $-2,25$ & -2 & 622 \\
\hline 2 & 500,00 & 500 & 628,85 & 629 & 371,15 & 372 & $-55,22$ & -55 & 317 \\
\hline 3 & 560,00 & 560 & 608,20 & 609 & 511,81 & 512 & $-20,66$ & -20 & 492 \\
\hline 4 & 662,00 & 662 & 624,34 & 625 & 699,66 & 700 & 16,14 & 17 & 717 \\
\hline 5 & 793,40 & 794 & 675,06 & 676 & 911,74 & 912 & 50,72 & 51 & 963 \\
\hline
\end{tabular}


Tabla 4. Cálculos para coeficiente de suavización $\alpha=0.5$

\begin{tabular}{|c|c|c|c|c|c|c|c|c|}
\hline \multicolumn{2}{|c|}{$\begin{array}{c}\text { PRONÓSTICO } \\
\text { SIMPLE }\end{array}$} & \multicolumn{2}{c|}{$\begin{array}{c}\text { PRONÓSTICO } \\
\text { DOBLE }\end{array}$} & \multicolumn{2}{c|}{ VALOR DE a } & \multicolumn{2}{c|}{ VALOR DE b } & PRON \\
\hline CÁLCULO & AJUSTE & CÁLCULO & AJUSTE & CÁLCULO & AJUSTE & CÁLCULO & AJUSTE & AJUSTE \\
\hline 777,50 & 778 & 795,03 & 796 & 759,97 & 760 & $-17,53$ & -17 & 743 \\
\hline 500,00 & 500 & 777,50 & 778 & 222,50 & 223 & $-277,50$ & -277 & -54 \\
\hline 600,00 & 600 & 688,75 & 689 & 511,25 & 512 & $-88,75$ & -88 & 424 \\
\hline 750,00 & 750 & 719,38 & 720 & 780,63 & 781 & 30,63 & 31 & 812 \\
\hline 925,00 & 925 & 822,19 & 823 & 1027,81 & 1028 & 102,81 & 103 & 1131 \\
\hline
\end{tabular}

Tabla 5. Cálculos para coeficiente de suavización $\alpha=0.7$

\begin{tabular}{|c|c|c|c|c|c|c|c|c|}
\hline \multicolumn{2}{|c|}{$\begin{array}{c}\text { PRONÓSTICO } \\
\text { SIMPLE }\end{array}$} & \multicolumn{2}{c|}{ PRONÓSTICO DOBLE } & VALOR DE a & & $\begin{array}{c}\text { VALOR } \\
\text { DE b }\end{array}$ & & PRON \\
\hline CÁLCULO & AJUSTE & CÁLCULO & AJUSTE & CÁLCULO & AJUSTE & CÁLCULO & AJUSTE & AJUSTE \\
\hline 744,65 & 745 & 784,38 & 785 & 704,92 & 705 & $-92,71$ & -92 & 613 \\
\hline 500,00 & 500 & 744,65 & 745 & 255,35 & 256 & $-570,85$ & -570 & -314 \\
\hline 640,00 & 640 & 671,40 & 672 & 608,61 & 609 & $-73,26$ & -73 & 536 \\
\hline 822,00 & 822 & 776,82 & 777 & 867,18 & 868 & 105,42 & 106 & 974 \\
\hline 1016,60 & 1017 & 944,67 & 945 & 1088,53 & 1089 & 167,85 & 168 & 1257 \\
\hline
\end{tabular}

Pronósticos alternos:

El método de Torres, trabaja tres planes alternos, (demanda alta, demanda baja y demanda constante), para lo cual usa los cálculos hechos basándose en los coeficientes de suavización $\alpha$ 0.3, 0.5 y 0.7.

Para hallar los valores de los planes alternos demanda alta, baja y constante, se debe buscar, para cada uno de los períodos, dentro de los datos ajustados (pronóstico simple, doble y pronóstico), el valor más alto, y se toma como el pronóstico alterno de demanda alta, el valor más bajo, que se refiere al pronóstico alterno de demanda baja y el valor más cercano al dato histórico, y se llama pronóstico alterno de demanda constante.
A continuación se pueden ver, los resultados buscados de las tres tablas anteriores correspondientes a los tres planes alternos, se debe recordar que la información de los planes alternos de producción, se elige de las tablas de los pronósticos ajustados de los diferentes coeficientes de suavización $\alpha$, para este ejemplo, $0.3,0.5$ y 0.7 :

\section{Tabla 6.}

\begin{tabular}{|c|c|c|}
\hline $\begin{array}{c}\text { Más cercano } \\
\text { al histórico }\end{array}$ & $\begin{array}{c}\text { Demanda } \\
\text { alta }\end{array}$ & $\begin{array}{c}\text { Demanda } \\
\text { baja }\end{array}$ \\
\hline $\mathrm{D} \rightarrow$ & $\mathrm{D} \uparrow$ & $\mathrm{D} \downarrow$ \\
\hline 613 & 785 & 613 \\
\hline 694 & 745 & 314 \\
\hline 672 & 672 & 492 \\
\hline 974 & 974 & 625 \\
\hline 1257 & 1257 & 676 \\
\hline
\end{tabular}


Aseguramiento del pronóstico:

Introduce un comportamiento heurístico, en el cual se determina el porcentaje de crecimiento o decrecimiento general de la demanda para el período siguiente. Torres propone que usando otro método se determine este porcentaje, como por ejemplo, el método Delphi ${ }^{17}$, que usa un panel de expertos en el que, a través de la experiencia ${ }^{18}$, se determina el porcentaje de crecimiento o decrecimiento general de la demanda.

\section{Demanda agregadda global:}

$$
\mathrm{DAG}_{\mathrm{t}}=\mathrm{D}_{\mathrm{t}} \times(1+\% \mathrm{P})
$$

Donde;

$\mathrm{D}_{\mathrm{t}}=$ dato más cercano al histórico en cada período

$\% \mathrm{P}=$ Crecimiento o decrecimiento ${ }^{19}$ general de la demanda para el siguiente período, si es decrecimiento debe usarse en la formula $-\%$ P.

\section{IÍndice de aseguramiento:}

$$
\mathrm{IA}_{\mathrm{t}+1}=\mathrm{X}_{\mathrm{t}+1} / \mathrm{X}_{\mathrm{t}}
$$

Donde;

$\mathrm{IA}_{\mathrm{t}}=$ índice de aseguramiento del comportamiento de la variable en el período $\mathrm{t}$ $\mathrm{X}_{\mathrm{t}}=$ demanda histórica o variable de entrada en el período $t$
Pronóstico final DAT:

$$
\mathrm{DAT}_{\mathrm{i}=1, \mathrm{n}}=\mathrm{DAG}_{\mathrm{t}} \times \mathrm{IA}_{\mathrm{t}}
$$

\section{Donde;}

$\operatorname{DAT}_{\mathrm{i}}=$ Demanda Agregada Total del período i, se refiere al valor pronosticado través del método, para los períodos siguientes.

$\mathrm{DAG}_{\mathrm{t}}=$ Demanda Agregada Global del período $t$

$\mathrm{IA}_{\mathrm{t}}=$ Índice de aseguramiento

Nótese que para este ejemplo se usó un índice de crecimiento de $0 \%$, porque con los datos, no es fácil determinar el comportamiento de la demanda en el próximo ciclo de 5 períodos.

Para ilustrar el ejemplo se tomó el plan alterno de producción de demanda constante igual a la demanda global, y se aplicó la formula del índice de aseguramiento para llegar al pronóstico final para los cinco períodos siguientes. A continuación se pueden ver los resultados de dichos cálculos:

Tabla 7.

\begin{tabular}{|c|c|c|}
\hline $\begin{array}{c}\text { Demanda } \\
\text { global }\end{array}$ & $\begin{array}{c}\text { Índice de } \\
\text { aseguramiento }\end{array}$ & $\begin{array}{c}\text { Pronóstico } \\
\text { final }\end{array}$ \\
\hline DAG & IA & DAT \\
\hline 613 & & 613 \\
\hline 694 & 1,4 & 972 \\
\hline 672 & 1,2857 & 864 \\
\hline 974 & 1,2222 & 1191 \\
\hline 1257 & 1,1818 & 1486 \\
\hline
\end{tabular}

17. Método Delphi. En el mismo texto en el que se describe el método de Torres se hace referencia a este método como el consenso de los criterios de personas expertas que sean capaces de inferir de manera sólida comportamientos futuros de variables tales como: demandas de productos, basados en conocimientos profundos del medio comercial y del manejo de variables macroeconómicas.

18. El método Delphi fue ideado por Olaf Helmer en 1966, si se desea ampliar el concepto del método Delphi, así como el resumen de su funcionamiento y las palabras claves, visite: http://papers.ssrn. com/sol3/papers.cfm?abstract_id=420040. También puede copiar el libro completo The Delphi Method: Techniques and Applications de la siguiente página web: http://www.is.njit.edu/pubs/delphibook/index.html.

19. \% $\mathrm{P}$, denota el crecimiento o disminución de la tendencia general de los datos; para el siguiente período el autor menciona este dato como el resultado del método Delphi. 
La formulación completa del método de Torres en la hoja de cálculo, puede accederse desde la siguiente página Web:

http://sigma.poligran.edu.co/politecnico/apoyo/cuadernos/cuadernoscont.htm

\section{ANÁLISIS DEL MÉTODO DETORRES}

En este aparte usamos ejemplos muy simples para examinar el comportamiento del método de Torres; es decir, creamos diferentes series de datos en los cuales se pudiera analizar la tendencia, la estaciona- lidad del método, y un aspecto muy importante que es la forma como el método trata de replicar el comportamiento del período tomado como base histórica, de manera que se pudieran ver algunas de sus bondades y sus limitaciones, para ello en el primer análisis se prueba el método usando series de demandas en seis (6), doce (12) y veinticuatro (24) períodos.

Algunos resultados se presentan a continuación:

Gráfica 1. Con 12 períodos

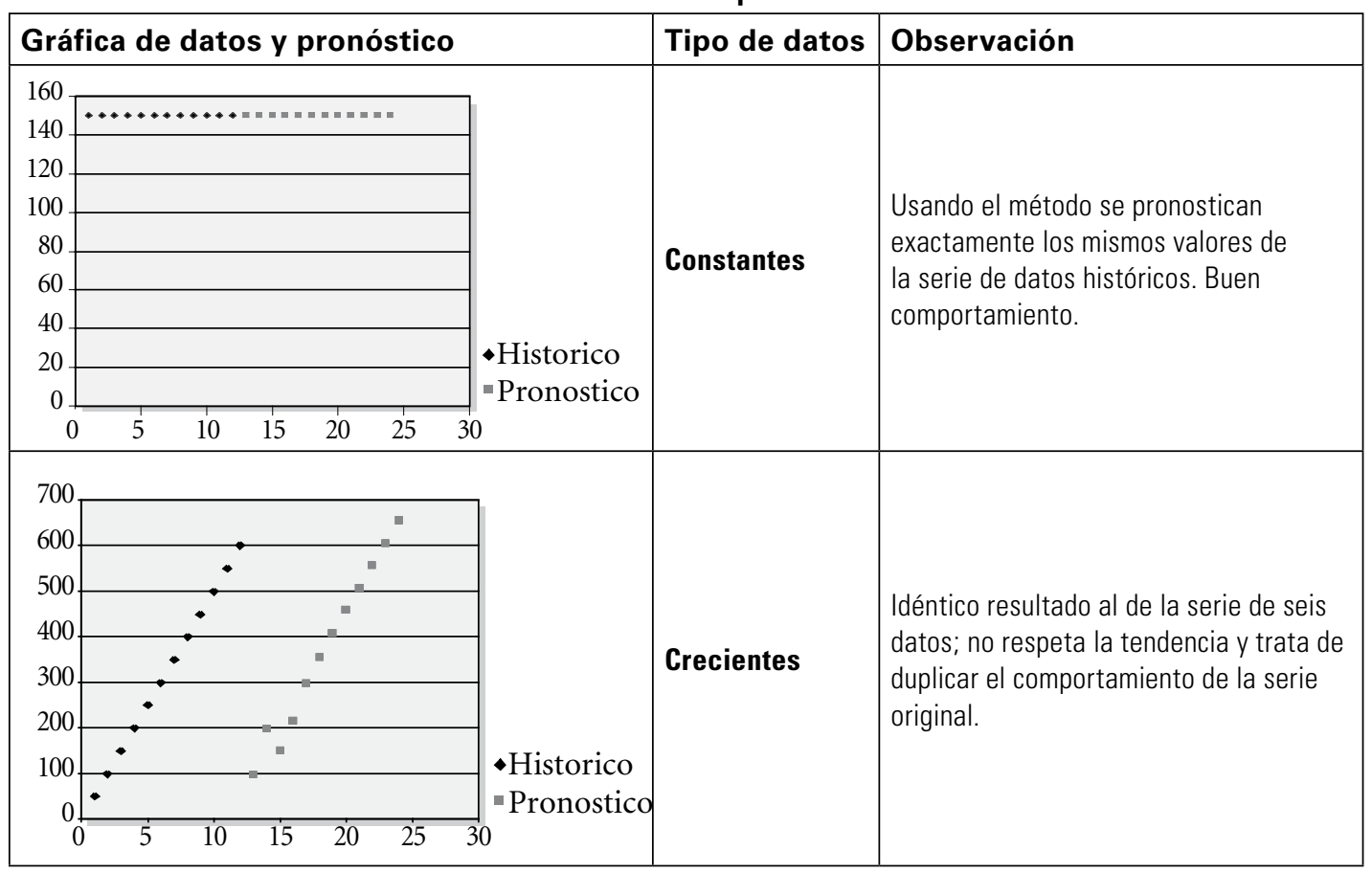




\begin{tabular}{|c|c|c|c|}
\hline Gráfica de datos y pronóstico & & Tipo de datos & Observación \\
\hline 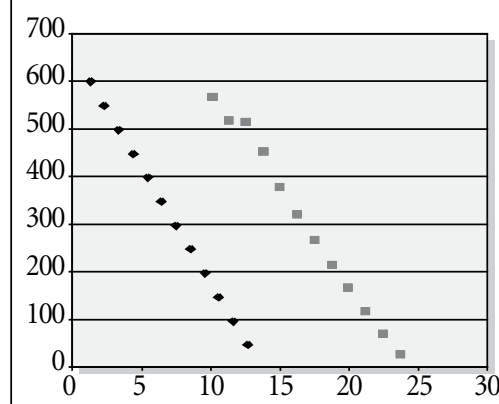 & $\begin{array}{l}- \text { Historico } \\
\text { - Pronostico } \\
0\end{array}$ & Decrecientes & $\begin{array}{l}\text { No respeta la tendencia de la variable. } \\
\text { Se comporta igual con una serie de seis } \\
\text { datos y con una serie de doce datos. }\end{array}$ \\
\hline 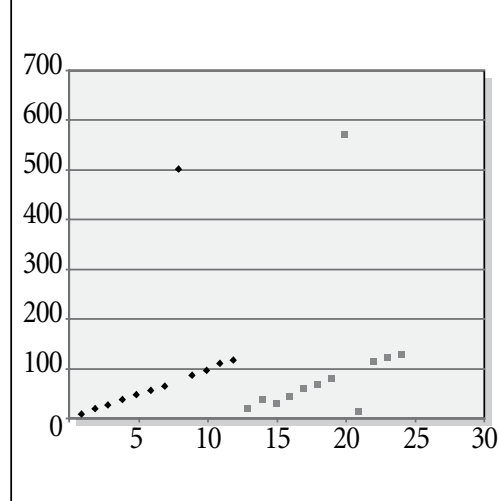 & $\begin{array}{l}- \text { Historico } \\
\text { - Pronostico }\end{array}$ & $\begin{array}{l}\text { Con } \\
\text { estacionalidad }\end{array}$ & $\begin{array}{l}\text { El resultado es muy satisfactorio, } \\
\text { porque en el mismo período en el } \\
\text { que se presenta el pico de los datos } \\
\text { históricos, en este caso en el período } \\
\text { 8, el pronóstico genera un pico muy } \\
\text { parecido en el mismo período 8. Buen } \\
\text { comportamiento frente a datos que } \\
\text { tengan estaciones marcadas. } \\
\text { El punto del pronóstico } 21 \text { extrañamente } \\
\text { toma un valor muy bajo, cuando los } \\
\text { datos históricos siguen una tendencia } \\
\text { clara. }\end{array}$ \\
\hline 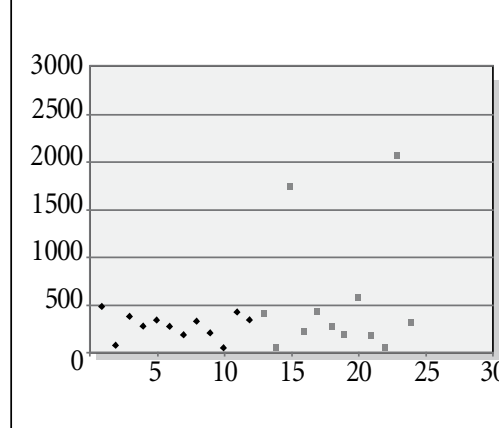 & $\begin{array}{l}\text { - Historico } \\
\text { - Pronostico }\end{array}$ & Aleatorios & $\begin{array}{l}\text { Es muy parecido al resultado } \\
\text { generado con la serie de seis datos, el } \\
\text { resultado también trata de replicar el } \\
\text { comportamiento de los datos originales } \\
\text { con incrementos o disminuciones } \\
\text { marcadas. Se pueden ver dos valores } \\
\text { fuera de los rangos esperados, períodos } \\
3 \text { y } 11 \text {, sin explicación aparente. } \\
\text { Extrañamente, en estos dos puntos el } \\
\text { pronóstico sube inesperadamente, y por } \\
\text { un rango demasiado elevado. }\end{array}$ \\
\hline
\end{tabular}

El modelo tiene una particularidad y es que los datos pronosticados respetan en gran medida la serie de los datos históricos; es decir, que si se incluye como información de entrada una serie de 100 datos, los pronósticos van a parecerse mucho a la serie histórica, así los cinco primeros datos pronosticados se parecerán más a los cinco primeros datos de la serie histórica y se pare- cerán menos a los cinco últimos datos de la serie histórica. Con éste análisis podemos observar que el método de pronostico le da más peso a los datos más alejados que a los más recientes, cuando se trata de pronosticar los siguientes. Esto puede ser bueno porque con pocos datos históricos se va a poder tener un pronóstico de la misma cantidad de datos, pero puede ser malo si se ve 
desde el punto de vista de la recopilación de datos, a largo plazo no va a poder simular comportamientos de ciclo.

Si desea ver los resultados del método usando diferentes series de datos como información de entrada, puede hacerlo desde la siguiente página Web:

http://sigma.poligran.edu.co/politecnico/apoyo/cuadernos/cuadernoscont. htm

\section{ANÁLISIS DE LAS PROYECCIONES CON MUCHOS Y POCOS DATOS}

El método de prueba que se usó es bastante simple; se tomó una colección de datos históricos reales de demanda y, usando el método de Torres, se comparó el resultado del mismo frente a los datos reales. Para ello no se usaron todos los datos como históricos, sino que se reservó el último grupo de datos para comparar la dispersión del método.

\subsection{PRUEBAUNO}

Los datos originales son 108 datos de demandas mensuales ${ }^{20}$, es decir, 9 años. Los datos tomados como históricos son 96 y los últimos doce datos se reservan para probar la precisión del método de Torres.

Los 96 datos históricos son los siguientes:

Tabla 8..

\begin{tabular}{|c|c|c|c|c|c|c|c|c|}
\hline Mes & Año 1 & Año 2 & Año 3 & Año 4 & Año 5 & Año 6 & Año 7 & Año 8 \\
\hline Enero & 1738 & 2224 & 2939 & 4009 & 5236 & 7242 & 10058 & 13959 \\
\hline Febrero & 1983 & 2459 & 3320 & 4577 & 6037 & 7955 & 10997 & 14873 \\
\hline Marzo & 2240 & 2657 & 3623 & 5039 & 6608 & 9149 & 12133 & 16568 \\
\hline Abril & 2138 & 2858 & 3765 & 4717 & 6426 & 8670 & 12182 & 16972 \\
\hline Mayo & 2330 & 2832 & 3812 & 4969 & 6614 & 9561 & 12916 & 17023 \\
\hline Junio & 2333 & 2782 & 4157 & 5283 & 6986 & 9636 & 13032 & 17237 \\
\hline Julio & 2320 & 3025 & 4100 & 5209 & 7017 & 9790 & 13379 & 18034 \\
\hline Agosto & 2364 & 3002 & 4135 & 5250 & 7322 & 10182 & 13955 & 17789 \\
\hline Septiembre & 2463 & 3151 & 4220 & 5597 & 7658 & 10441 & 14185 & 18168 \\
\hline Octubre & 2579 & 3283 & 4313 & 5449 & 7661 & 11184 & 14984 & 19031 \\
\hline Noviembre & 2545 & 3355 & 4440 & 5704 & 8070 & 11562 & 14825 & 18234 \\
\hline Diciembre & 4091 & 5296 & 6909 & 9154 & 10877 & 14628 & 18964 & 24379 \\
\hline \hline
\end{tabular}

20. Datos de demandas históricas tomados de Vélez, Pareja. Decisiones empresariales bajo incertidumbre. Bogotá: Norma. 2004 También puede accederlos desde: http://www.poligran.edu.co/decisiones 


\section{Gráfica 2.}

Demandas

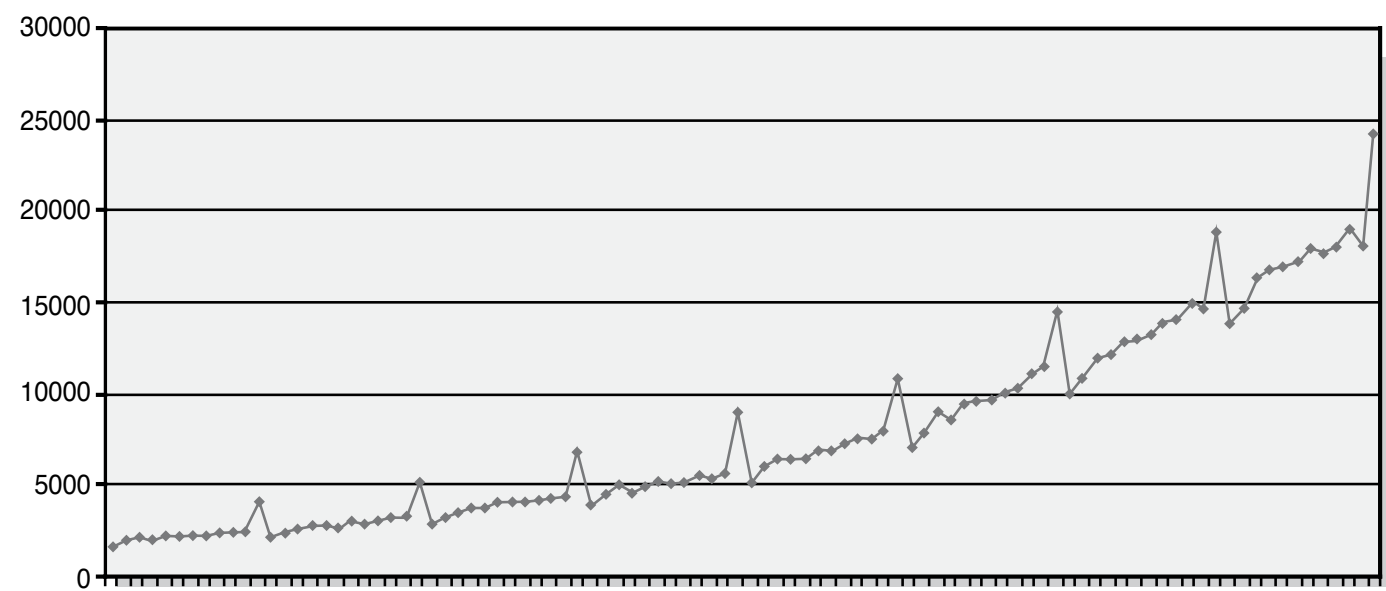

EM M J S NEM M J SNEM M J S NEM M J S NEM M J S NEM M J S NEM M J S NEM M J S N

En la gráfica se puede ver el comportamiento de los datos históricos de la demanda, para los 96 datos históricos, que corresponden a los 8 primeros años; los datos restantes, se reservarán para medir la precisión del método de Torres.
A continuación se muestran los resultados que arrojó el método de Torres, usando los ocho años de demandas históricas:

Tabla 9.

\begin{tabular}{|c|c|c|c|c|}
\hline Período & $\begin{array}{c}\text { Demanda } \\
\text { real }\end{array}$ & $\begin{array}{c}\text { Método Torres } \\
\text { usando todos los datos }\end{array}$ & $\begin{array}{c}\text { Diferencia en } \\
\text { unidades }\end{array}$ & $\begin{array}{c}\text { Diferencia al } \\
\text { cuadrado }\end{array}$ \\
\hline Enero & 17.176 & 2.291 & 14.885 & 221.563 .225 \\
\hline Febrero & 18.327 & 2.750 & 15.577 & 242.642 .929 \\
\hline Marzo & 21.819 & 2.665 & 19.154 & 366.875 .716 \\
\hline Abril & 19.627 & 2.474 & 17.153 & 294.225 .409 \\
\hline Mayo & 20.969 & 2.905 & 18.064 & 326.308 .096 \\
\hline Junio & 22.046 & 2.889 & 19.157 & 366.990 .649 \\
\hline Julio & 22.068 & 2.787 & 19.281 & 371.756 .961 \\
\hline Agosto & 21.949 & 2.897 & 19.052 & 362.978 .704 \\
\hline Septiembre & 22.630 & 3.064 & 19.566 & 382.828 .356 \\
\hline Octubre & 23.609 & 3.195 & 20.414 & 416.731 .396 \\
\hline Noviembre & 22.573 & 3.028 & 19.545 & 382.007 .025 \\
\hline Diciémbre & 29.583 & 5.115 & 24.468 & 598.683 .024 \\
\hline Totales & 262.376 & 36.060 & 226.316 & 4.333 .591 .490 \\
\hline
\end{tabular}


El primer problema encontrado, es el desfase tan grande entre los datos pronosticados y los datos reales de la variable. Se ve claramente que los datos pronosticados son muy parecidos a los valores históricos del año uno y muy diferentes o alejados de los correspondientes al año ocho. Este resultado era predecible, gracias al análisis del método y al comportamiento visto en las gráficas que mostraban claramente que el método trata de replicar los valores iniciales de la serie.

En el primer análisis de las gráficas se mencionó, que cuando había estacionali- dad, el método se comportaba bien; pero, en este caso, el número de datos es muy grande, 96 datos. Y por eso, los resultados del pronóstico tratan de parecerse a los del primer año, respetando de algún modo el comportamiento de picos en los mismos períodos de los datos históricos, pero muy lejos de los datos reales porque difieren mucho de los del año 8, y no respetan la tendencia general de los datos históricos. En la siguiente gráfica se puede ver este comportamiento:

\section{Gráfica 3.}

Usando 96 Datos

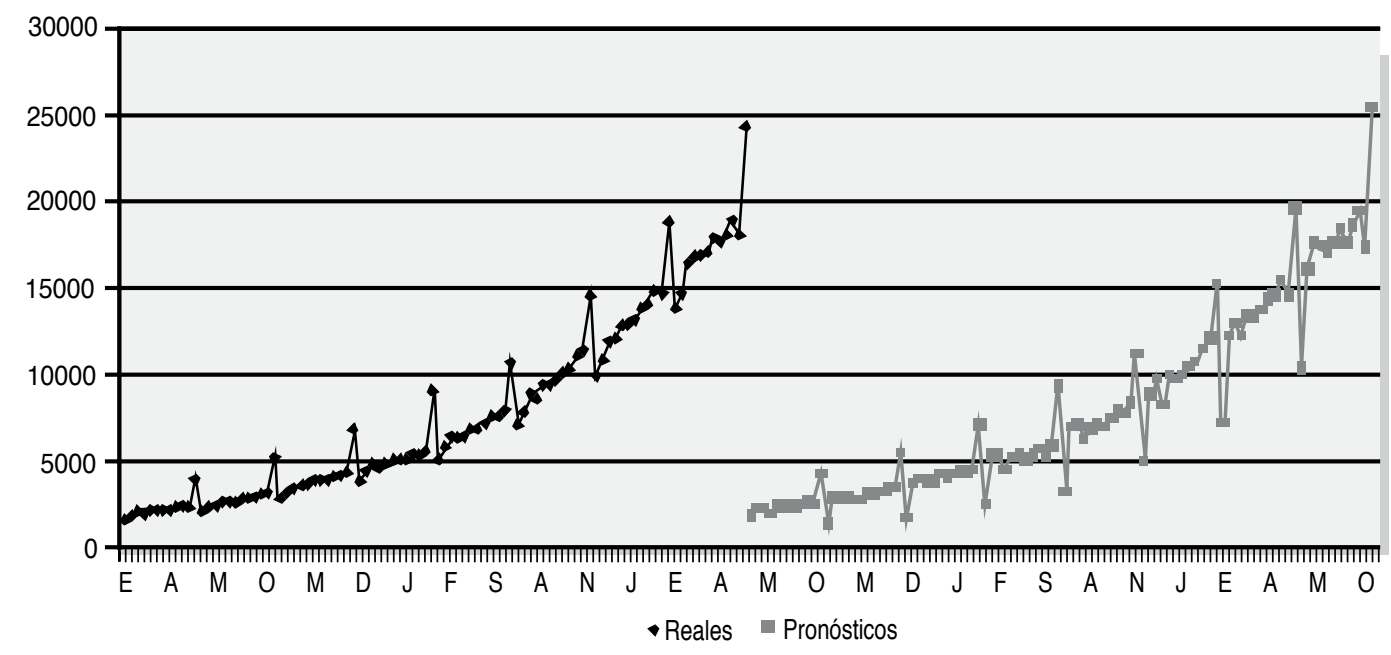

Se usaron los últimos doce datos, es de Torres se obtuvieron unos resultados decir, el último año de los datos históri- mucho más satisfactorios. A continuación cos como datos de entrada, y del método se muestran los resultados obtenidos:

Tabla 10.

\begin{tabular}{|c|c|c|c|c|}
\hline Período & $\begin{array}{c}\text { Demanda } \\
\text { real }\end{array}$ & $\begin{array}{c}\text { Método de Torres usando } \\
\text { el último período }\end{array}$ & $\begin{array}{c}\text { Diferencia en } \\
\text { unidades }\end{array}$ & $\begin{array}{c}\text { Diferencia al } \\
\text { cuadrado }\end{array}$ \\
\hline Enero & 17.176 & 14.837 & 2.339 & 5.470 .921 \\
\hline Febrero & 18.327 & 15.910 & 2.417 & 5.841 .889 \\
\hline Marzo & 21.819 & 16.606 & 5.213 & 27.175 .369 \\
\hline Abril & 19.627 & 17.465 & 2.162 & 4.674 .244 \\
\hline Mayo & 20.969 & 17.330 & 3.639 & 13.242 .321 \\
\hline
\end{tabular}




\begin{tabular}{|c|c|c|c|c|}
\hline Período & $\begin{array}{c}\text { Demanda } \\
\text { real }\end{array}$ & $\begin{array}{c}\text { Método de Torres usando } \\
\text { el último período }\end{array}$ & $\begin{array}{c}\text { Diferencia en } \\
\text { unidades }\end{array}$ & $\begin{array}{c}\text { Diferencia al } \\
\text { cuadrado }\end{array}$ \\
\hline Junio & 22.046 & 17.689 & 4.357 & 18.983 .449 \\
\hline Julio & 22.068 & 18.516 & 3.552 & 12.616 .704 \\
\hline Agosto & 21.949 & 17.526 & 4.423 & 19.562 .929 \\
\hline Septiembre & 22.630 & 18.645 & 3.985 & 15.880 .225 \\
\hline Octubre & 23.609 & 19.443 & 4.166 & 17.355 .556 \\
\hline Noviembre & 22.573 & 17.286 & 5.287 & 27.952 .369 \\
\hline Diciémbre & 29.583 & 25.386 & 4.197 & 17.614 .809 \\
\hline Totales & 262.376 & 216.639 & 45.737 & 186.370 .785 \\
\hline
\end{tabular}

A continuación puede observarse año. Se puede ver claramente que el proel comportamiento del pronóstico que nóstico mejora notablemente usando usa sólo los datos del último período, es solo 12 datos frente al de los 96 datos decir los datos de los meses del último históricos.

Gráfica 4

Usando últimos 12 Datos

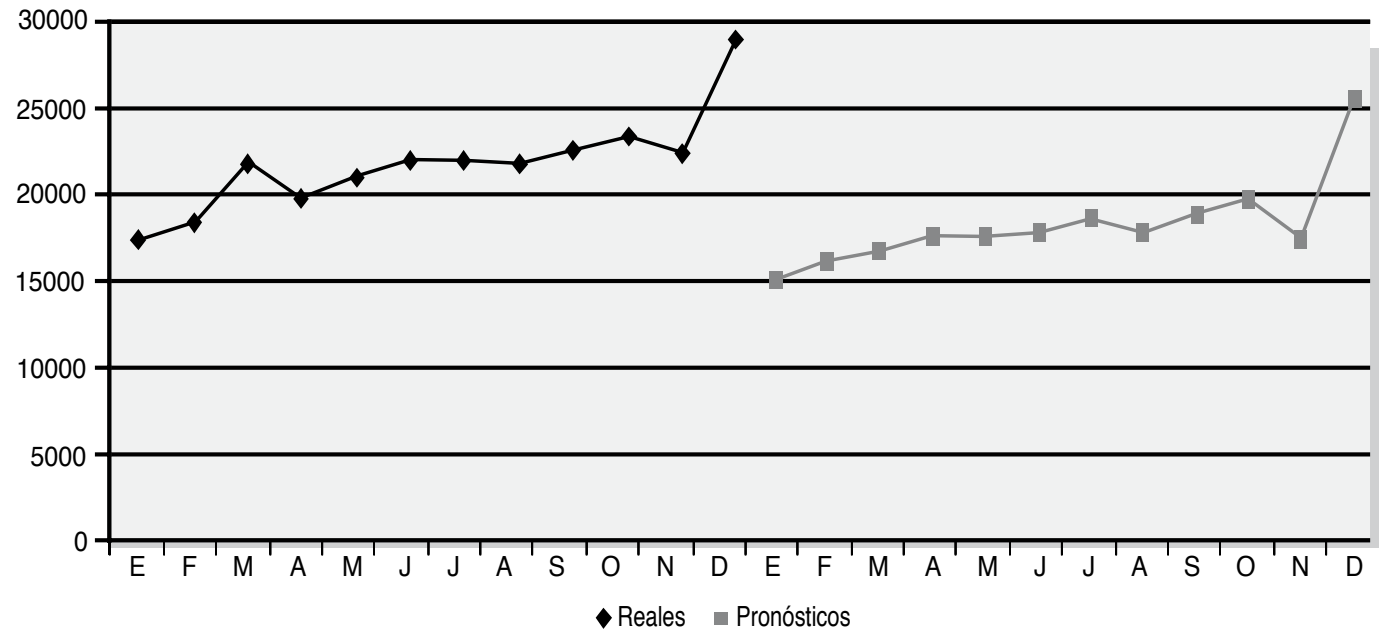

Usando los resultados del método de Dispersión en diferencias al cuadrado

Torres con los últimos 12 datos como datos

Total demanda real: 262.376

históricos y los valores reales de la serie de

Total diferencia en unidades usando todos datos, se puede observar en este momento, los 96 datos: 226.316

una diferencia del $\mathbf{2 1 . 1 1 \%}$ con respecto al total

Total diferencia al cuadrado: 4.333’591.490 de las demandas, calculada sobre el total de las demandas, para determinar en general, qué tan diferentes son los totales pronosticados $y$ el total de las demandas reales del año 9.

Total demanda real: 262.376

Total diferencia en unidades usando los últimos 12 datos: 45.737

Total diferencia al cuadrado: 186’370.785 
Claramente se puede notar que es mucho menor la diferencia de cuadrados al usar los últimos 12 datos y no los 96 datos históricos. Se puede establecer que es mejor usar los datos más recientes, porque el método de Torres trata de replicar el comportamiento de toda la serie de datos, y si se toman datos muy diferentes a los más recientes, el resultado del pronóstico va a empezar muy parecido a los primeros datos históricos.

\section{ANÁLISIS DEL FACTOR SUBJETIVO DE CRECIMIENTO O DECRECIMIENTO USADO EN EL MÉTODO}

El método de Torres menciona como información de entrada, el factor de crecimiento o disminución ${ }^{21}$ de la serie, -así lo llama en su texto-, a partir del cual surge un segundo problema. Dicho más claramente, ¿cuál debería de la manera de involucrar este factor en el modelo, pero basados en la serie de datos? $2 i ¿$ ??? Es una manera de volver el factor subjetivo del método de Torres, en un factor que pueda abstraerse del comportamiento de la serie de datos.

Se trata de determinar, usando alguna técnica, si la serie general de los datos frente al período va a crecer o a decrecer, y mostrarlo en forma de porcentaje. La solución que saltó a la vista fue analizar la serie de datos y determinar frente a los ciclos o períodos, en qué porcentaje estaba cambiando la serie; si es positiva, crece y si es negativa, decrece.

Luego se trabajó con el promedio de estas variaciones para minimizar el error, y finalmente, se analizó la serie de las variaciones y se extrapolaron, usando para ello las líneas de tendencia, y escogiendo la que tuviera el mayor $\mathrm{R}^{2}$ (error al cuadrado) ${ }^{22}$. Para este fin, se realizó un análisis por separado de la serie de datos de las variaciones que sufrían los datos históricos período a período, tratando de darle objetividad a este factor de naturaleza subjetiva.

Para este ejemplo en particular, se midieron las variaciones en cada uno de los años, de modo que se tienen cuatro pruebas diferentes:

- Sin variación

- Usando la última variación

- Usando el promedio de las variaciones de todos los años

- Usando la extrapolación que tenga mayor $\mathrm{R}^{2}$

A continuación se pueden observar los promedios de los datos históricos:

Tabla 11.

\begin{tabular}{|l|r|}
\hline \multicolumn{2}{|c|}{ Promedios } \\
\hline 1975 & 2427,00 \\
\hline 1976 & 3077,00 \\
\hline 1977 & 4144,42 \\
\hline 1978 & 5413,08 \\
\hline 1979 & 7209,33 \\
\hline 1980 & 10000,00 \\
\hline 1981 & 13467,50 \\
\hline 1982 & 17688,92 \\
\hline
\end{tabular}

Como puede verse, cada uno de los períodos sufre una variación frente al período siguiente, que en este caso en particular, es un año, es decir 12 datos. lo que se hizo fue calcular en cuánto aumenta o disminuye el promedio general del período.

21. Se refiere al mismo valor \%P, descrito en el método de Torres. Para ampliar este concepto ver: TORRES ACOSTA, Jairo Humberto. Op.cit. Pág. 74.

22. $\mathrm{R}^{2}$, se refiere al nivel de ajuste de la línea de tendencia a la serie de datos, mientras más cercano a uno, mayor es el ajuste de la línea de tendencia. 
De esta manera, si es positivo el cambio, se supone un crecimiento en la serie y si es negativo, se supone que la serie decrece. A continuación puede verse la variación, ya sea positiva o negativa para cada período:

Tabla 12.

\begin{tabular}{|l|r|}
\hline Año & $\begin{array}{c}\text { Cambio en el } \\
\text { promedio }\end{array}$ \\
\hline $1975-1976$ & $26,78 \%$ \\
\hline $1976-1977$ & $34,69 \%$ \\
\hline $1977-1978$ & $30,61 \%$ \\
\hline $1978-1979$ & $33,18 \%$ \\
\hline $1979-1980$ & $38,71 \%$ \\
\hline $1980-1981$ & $34,68 \%$ \\
\hline $1981-1982$ & $31,35 \%$ \\
\hline Promedio & $32.85 \%$ \\
\hline
\end{tabular}

Para analizar el comportamiento general de la variable y saber en qué grado crece o decrece, lo que se hizo fue, crear una serie con los valores de las variaciones de los períodos, con la ayuda de la hoja de cálculo, graficarlas, agregarles líneas de tendencia y registrar tanto el valor del $\mathrm{R}^{2}$, el valor de la función que agrupa a los datos de la serie, como el valor esperado para el siguiente período, extrapolando dicha serie de variaciones en un período hacia adelante para predecir la variación que va a tener la variable de la demanda en el período siguiente. A continuación puede verse el análisis completo de las series de crecimientos extrapoladas con las diferentes líneas de tendencia:

- Lineal

- Exponencial

- Logarítmica

- Potencial

- Polinomial grado 2

- Polinomial grado 3
Gráficas 5

Tendencia Lineal

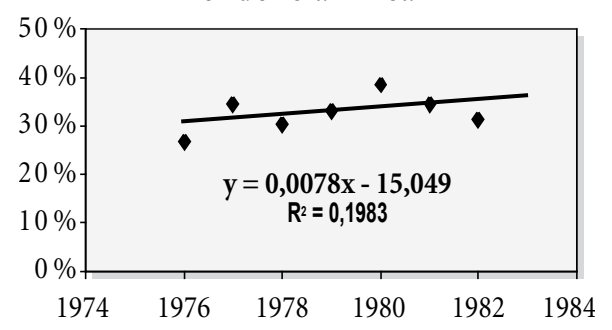

Tendencia Exponencial

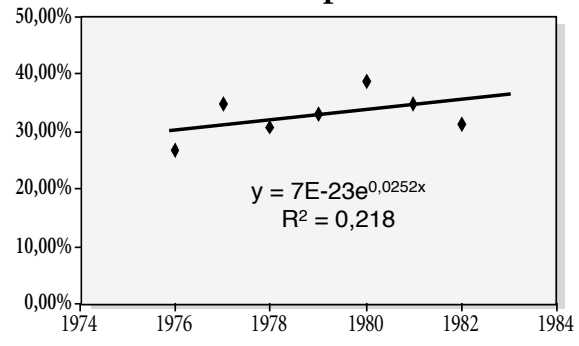

Tendencia Logaritmica

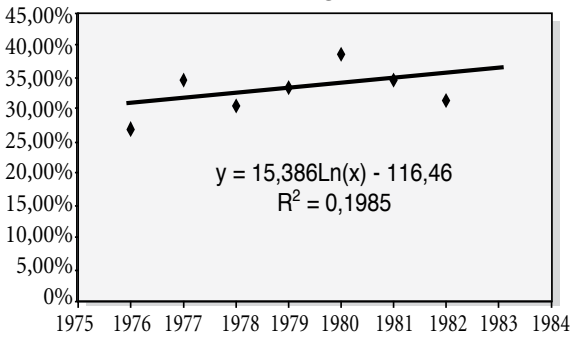

Tendencia Potencial

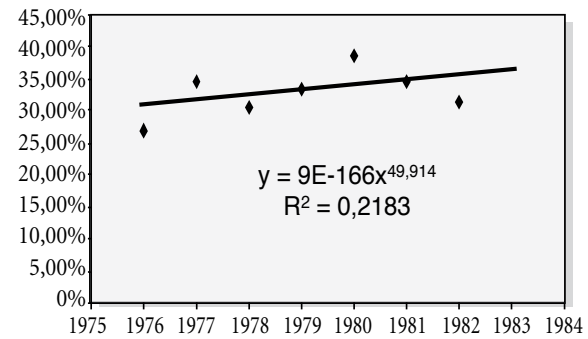

Polinomial 2

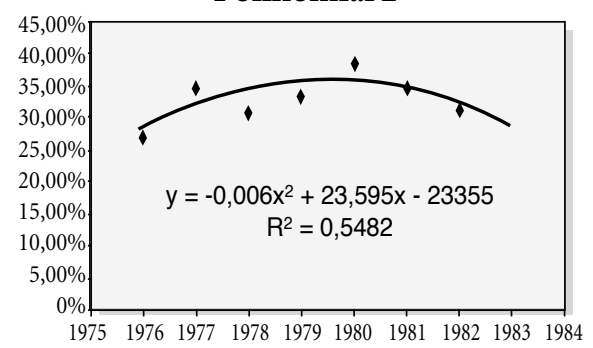




\section{Polinomial 3}

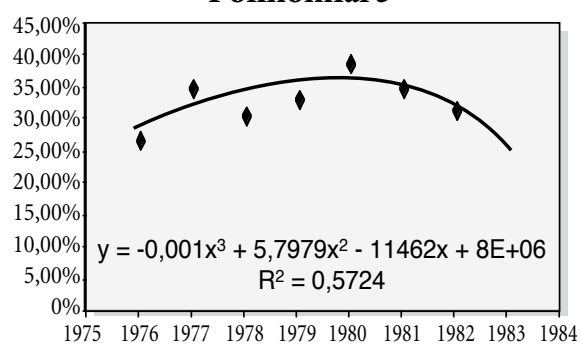

A partir de este análisis, puede afirmarse, que el mejor ajuste se logra con una línea de tendencia polinomial grado 3 , con la cual se obtiene un dato esperado de crecimiento del $25 \%$. A continuación se resumen los resultados del análisis de tendencias de las variaciones de cada uno de los períodos y de los valores esperados:

Tabla 13.

\begin{tabular}{|l|c|c|}
\hline Tendencias & R2 & Variación \\
\hline Lineal & 0,1983 & $36 \%$ \\
\hline Exponencial & 0,2180 & $36 \%$ \\
\hline Logarítmica & 0,1985 & $36 \%$ \\
\hline Potencial & 0,2183 & $36 \%$ \\
\hline Polinomial 2 & $\mathbf{0 , 5 4 8 2}$ & $\mathbf{2 9} \%$ \\
\hline Polinomial 3 & $\mathbf{0 , 5 7 2 4}$ & $\mathbf{2 5} \%$ \\
\hline
\end{tabular}

Con estos resultados, es pertinente el método de Torres usando como \% $\mathrm{P}$ (crecimiento o disminución general de la demanda para el siguiente período, si es disminución debe usarse en la formula $\% \mathrm{P})$, los siguientes:

- El resultado del mayor $\mathrm{R}^{2}$, es decir $\% \mathrm{P}=$ $25 \%$

- El último valor de variación, es decir, \%P $=31.35 \%$
- El valor de las variaciones generales, (promedio de las variaciones), es decir, $\% \mathrm{P}=$ $32.86 \%$

- Y por supuesto, sin usar factor de crecimiento, es decir $\% \mathrm{P}=0 \%$

El mejor resultado se logró, usando el 25\% como factor de crecimiento. Este resultado lo produjo el análisis de las líneas de tendencia, con el ajuste polinomial grado 3 .

Con este método de prueba, es decir, midiendo el menor error cuadrático, partiendo de la diferencia entre el dato pronosticado menos la demanda, y ese resultado elevado al cuadrado. La suma de todos los errores al cuadrado RSE ${ }^{23}$, puede determinar cuál estuvo más cerca del dato real. Los valores de las diferencias al cuadrado se muestran a continuación, junto con los valores de los pronósticos, usando las diferentes variaciones:

\section{PRONÓSTICOS}

Con base en el análisis presentado en el numeral 5, en el que se estableció la conveniencia de usar los datos del período más reciente, (12 últimos datos), en lugar de usar los 96 datos históricos, se ejecutó el método de Torres, pero variando los valores de $\% \mathrm{P}$, obtenidos con las variaciones promedio, y la variación con mayor $\mathrm{R}^{2}$. A continuación se presentan los resultados del método de Torres con los últimos datos para diferentes valores de \%P:

23. RSE hace referencia al valor de la suma de los errores elevados al cuadrado. Para éste caso, llámese error al valor de diferencia entre el dato pronosticado y el dato real. Si se desea ampliar el concepto de RSE diríjase a MAKRIDAKIS, Spyros.,Op.cit. Pág. 75 a 81 
Tabla 14.

\begin{tabular}{|c|c|c|c|c|c|}
\hline \multirow{2}{*}{ Período } & \multirow{2}{*}{$\begin{array}{c}\text { Demanda } \\
\text { real }\end{array}$} & Sin variación & $\begin{array}{c}\text { Última } \\
\text { variación }\end{array}$ & $\begin{array}{c}\text { Variaciones } \\
\text { Promedio }\end{array}$ & Mayor $\mathbf{R}^{\mathbf{2}}$ \\
\cline { 3 - 6 } & Crecimiento & $\begin{array}{c}\text { Torres con } 31,35 \% \\
\text { Crecimiento }\end{array}$ & $\begin{array}{c}\text { Torres con 32,86\% } \\
\text { Crecimiento }\end{array}$ & $\begin{array}{c}\text { Torres con 25\% } \\
\text { Crecimiento }\end{array}$ \\
\hline E & 17.176 & 14.837 & 19.489 & 19.713 & 18.547 \\
\hline F & 18.327 & 15.910 & 20.899 & 21.139 & 19.888 \\
\hline M & 21.819 & 16.606 & 21.813 & 22.064 & 20.758 \\
\hline A & 19.627 & 17.465 & 22.941 & 23.205 & 21.832 \\
\hline M & 20.969 & 17.330 & 22.764 & 23.025 & 21.663 \\
\hline J & 22.046 & 17.689 & 23.235 & 23.502 & 22.112 \\
\hline J & 22.068 & 18.516 & 24.321 & 24.601 & 23.145 \\
\hline A & 21.949 & 17.526 & 23.020 & 23.286 & 21.908 \\
\hline S & 22.630 & 18.645 & 24.491 & 24.772 & 23.307 \\
\hline D & 23.609 & 19.443 & 25.539 & 25.833 & 24.305 \\
\hline N & 22.573 & 17.286 & 22.705 & 22.967 & 21.608 \\
\hline D & 29.583 & 25.386 & 33.345 & 33.729 & 31.733 \\
\hline Totales & $\mathbf{2 6 2 . 3 7 6}$ & $\mathbf{2 1 6 . 6 3 9}$ & $\mathbf{2 8 4 . 5 6 2}$ & $\mathbf{2 8 7 . 8 3 6}$ & $\mathbf{2 7 0 . 8 0 6}$ \\
\hline & & & & \\
\hline
\end{tabular}

Tabla 15. ERRORES ELEVADOS AL CUADRADO

\begin{tabular}{|c|c|c|c|c|}
\hline \multirow{2}{*}{ Período } & Sin variación & $\begin{array}{c}\text { Última } \\
\text { variación }\end{array}$ & $\begin{array}{c}\text { Variaciones } \\
\text { promedio }\end{array}$ & Mayor $\mathbf{R}^{\mathbf{2}}$ \\
\cline { 2 - 5 } & $\begin{array}{c}\text { Torres con 0\% } \\
\text { Crecimiento }\end{array}$ & $\begin{array}{c}\text { Torres con 31,35\% } \\
\text { Crecimiento }\end{array}$ & $\begin{array}{c}\text { Torres con 32,86\% } \\
\text { Crecimiento }\end{array}$ & $\begin{array}{c}\text { Torres con 25\% } \\
\text { Crecimiento }\end{array}$ \\
\hline E & 5.470 .921 & 5.349 .969 & 6.436 .369 & 1.879 .641 \\
\hline F & 5.841 .889 & 6.615 .184 & 7.907 .344 & 2.436 .721 \\
\hline M & 27.175 .369 & 36 & 60.025 & 1.125 .721 \\
\hline A & 4.674 .244 & 10.982 .596 & 12.802 .084 & 4.862 .025 \\
\hline M & 13.242 .321 & 3.222 .025 & 4.227 .136 & 481.636 \\
\hline J & 18.983 .449 & 1.413 .721 & 2.119 .936 & 4.356 \\
\hline J & 12.616 .704 & 5.076 .009 & 6.416 .089 & 1.159 .929 \\
\hline A & 19.562 .929 & 1.147 .041 & 1.787 .569 & 1.681 \\
\hline S & 15.880 .225 & 3.463 .321 & 4.588 .164 & 458.329 \\
\hline D & 17.355 .556 & 3.724 .900 & 4.946 .176 & 484.416 \\
\hline N & 27.952 .369 & 17.424 & 155.236 & 931.225 \\
\hline D & 17.614 .809 & 14.152 .644 & 17.189 .316 & 4.622 .500 \\
\hline Totales & $\mathbf{1 8 6 . 3 7 0 . 7 8 5}$ & $\mathbf{5 5 . 1 6 4 . 8 7 0}$ & $\mathbf{6 8 . 6 3 5 . 4 4 4}$ & $\mathbf{1 8 . 4 4 8 . 1 8 0}$ \\
\hline
\end{tabular}

Como se puede apreciar, el menor grado de dispersión al sumar las demandas reales del año 9 y los datos pronosticados del mismo año, lo presenta el factor de crecimiento aportado por el valor esperado de la serie de datos de los crecimientos con mayor $\mathrm{R}^{2}$. En este caso se puede mencionar que el resultado de los pronósticos del método de Torres, sí se ve mejorado, cuando se usa el método del mayor $\mathrm{R}^{2}$ para determinar el valor de $\% \mathrm{P}$. 
6.1.PRUEBADOS

Para realizar una segunda prueba se eligió una nueva serie de datos, que representan el consumo promedio de kilowatios de energía eléctrica en Colombia ${ }^{24}$ en los últimos seis años; es decir, una serie de 72 datos. A continuación pueden verse los datos originales:

Tabla 16.

\begin{tabular}{|c|c|c|c|c|c|c|c|}
\hline \multicolumn{7}{|c|}{ Demanda energía promedio } \\
\hline Período & Mes & $\mathbf{1 9 9 9}$ & $\mathbf{2 0 0 0}$ & $\mathbf{2 0 0 1}$ & $\mathbf{2 0 0 2}$ & $\mathbf{2 0 0 3}$ & $\mathbf{2 0 0 4}$ \\
\hline ENERO & $\mathrm{E}$ & 117,8 & 114,6 & 119,1 & 122,3 & 127,8 & 128,6 \\
\hline FEBRERO & $\mathrm{F}$ & 120,1 & 119,7 & 122,3 & 127,2 & 130,9 & 134,0 \\
\hline MARZO & $\mathrm{M}$ & 119,0 & 119,1 & 122,5 & 126,3 & 131,4 & 135,3 \\
\hline ABRIL & $\mathrm{A}$ & 119,1 & 119,4 & 122,4 & 124,8 & 129,5 & 133,0 \\
\hline MAYO & $\mathrm{M}$ & 119,2 & 119,9 & 122,7 & 127,2 & 130,1 & 133,2 \\
\hline JUNIO & $\mathrm{J}$ & 118,0 & 120,7 & 122,0 & 125,4 & 128,0 & 133,6 \\
\hline JULIO & $\mathrm{JI}$ & 118,4 & 121,7 & 122,6 & 125,4 & 129,8 & 131,8 \\
\hline AGOSTO & $\mathrm{A}$ & 118,9 & 121,8 & 126,3 & 127,9 & 131,5 & 135,1 \\
\hline SEPTIEMBRE & $\mathrm{S}$ & 118,4 & 121,3 & 125,6 & 129,1 & 131,7 & 133,5 \\
\hline OCTUBRE & $\mathrm{D}$ & 119,9 & 121,5 & 126,2 & 129,0 & 131,8 & 133,8 \\
\hline NOVIEMBRE & $\mathrm{N}$ & 121,0 & 123,6 & 124,7 & 130,1 & 133,5 & 135,1 \\
\hline DICIEMBRE & $\mathrm{D}$ & 118,8 & 124,9 & 125,3 & 130,1 & 132,4 & 134,9 \\
\hline
\end{tabular}

Gráfica 6

Demanda de energía en GWh

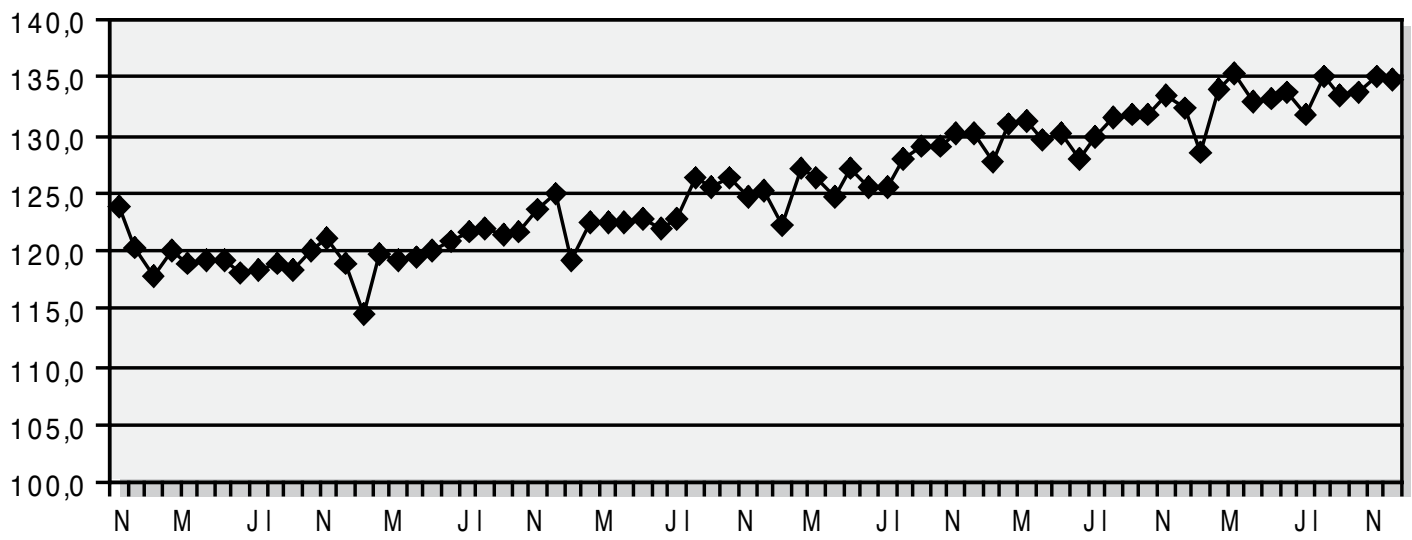

En la gráfica anterior se puede observar el medir la eficiencia del método de Torres. comportamiento del consumo nacional de enerEl siguiente es el análisis del factor de gía eléctrica, desde el año 1998 hasta el año 2003, crecimiento esperado de la serie, junto con la información real del año 2004, se reserva para las variaciones para cada período:

24. Consumo promedio de kilowatios de energía eléctrica por año, Fuente: Departamento Nacional de Planeación (DNP), puede accederse a esta serie de datos desde la página Web: http://www.dnp.gov.co, en el apartado de Estadísticas 
Tabla 17.

\begin{tabular}{|l|r|r|}
\hline \multicolumn{2}{|c|}{ Promedios } & $\begin{array}{c}\text { Cambio en el } \\
\text { promedio }\end{array}$ \\
\hline 1999 & 118,88 & \\
\hline 2000 & 119,96 & $0,91 \%$ \\
\hline 2001 & 123,36 & $2,83 \%$ \\
\hline 2002 & 126,21 & $2,31 \%$ \\
\hline 2003 & 130,21 & $3,17 \%$ \\
\hline 2004 & 133,15 & $2,26 \%$ \\
\hline Promedio & 125.295 & $2.296 \%$ \\
\hline
\end{tabular}

A continuación puede verse el análisis completo de las series de crecimientos extrapoladas con las diferentes líneas de tendencia:

Gráficas 8.

Tendencia Lineal

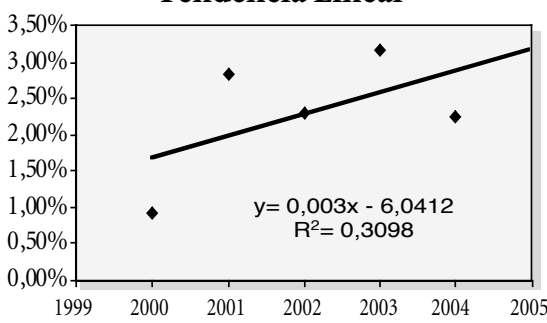

Tendencia Exponencial

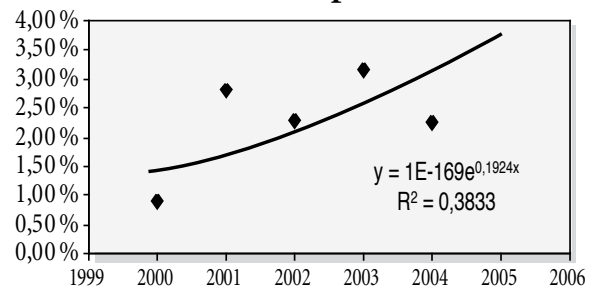

Tendencia Logarítmica

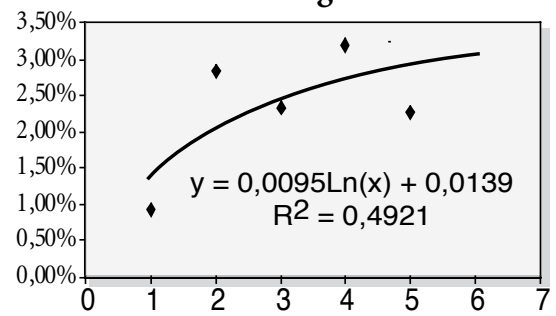

Tendencia Potencial

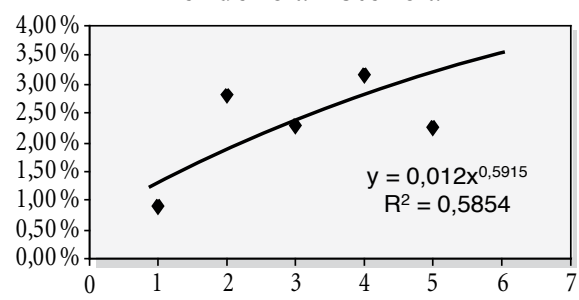

Polinomial 2

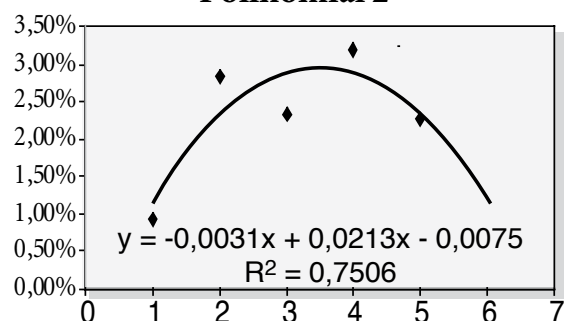

Polinomial 3

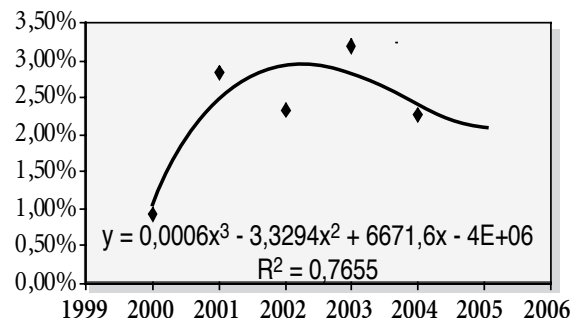

A continuación se resumen los resultados del análisis de tendencia de las variaciones de cada uno de los períodos y los valores esperados:

Tabla 18.

\begin{tabular}{|l|c|c|}
\hline \multicolumn{1}{|c|}{ Tendencias } & $\mathbf{R}^{\mathbf{2}}$ & $\begin{array}{c}\text { Cambio en } \\
\text { el promedio }\end{array}$ \\
\hline Lineal & 0,3098 & $3,2 \%$ \\
\hline Exponencial & 0,3833 & $3,8 \%$ \\
\hline Logarítmica & 0,4921 & $3,2 \%$ \\
\hline Potencial & 0.5854 & $3.5 \%$ \\
\hline Polinomial 2 & $\mathbf{0 , 7 5 0 6}$ & $\mathbf{1 , 4 \%}$ \\
\hline Polinomial 3 & $\mathbf{0 , 7 6 5 5}$ & $\mathbf{2 , 3} \%$ \\
\hline
\end{tabular}

Usando el Método de Torres para pronosticar las demandas de los siguientes 12 períodos, se tomaron como base histórica los últimos 12 meses; es decir, el año más reciente, con diferentes factores de crecimiento \% $\mathrm{P}$, los resultados son: 
Tabla 19.

\begin{tabular}{|c|c|c|c|c|c|}
\hline \multirow{2}{*}{ Período } & \multirow{2}{*}{$\begin{array}{c}\text { Demanda } \\
\text { real }\end{array}$} & Sin variación & $\begin{array}{c}\text { Última } \\
\text { variación }\end{array}$ & $\begin{array}{c}\text { Variaciones } \\
\text { Promedio }\end{array}$ & ${\text { Mayor } \mathbf{R}^{\mathbf{2}}}$ \\
\cline { 3 - 6 } & $\begin{array}{c}\text { Torres con 0\% } \\
\text { Crecimiento }\end{array}$ & $\begin{array}{c}\text { Torres con 2,26\% } \\
\text { Crecimiento }\end{array}$ & $\begin{array}{c}\text { Torres con 2,296\% } \\
\text { Crecimiento }\end{array}$ & $\begin{array}{c}\text { Torres con 2,3\% } \\
\text { Crecimiento }\end{array}$ \\
\hline E & 128,60 & 131,00 & 134,00 & 135,00 & 135,00 \\
\hline F & 134,02 & 138,61 & 142,77 & 142,77 & 142,77 \\
\hline M & 135,25 & 134,22 & 138,26 & 138,26 & 138,26 \\
\hline A & 133,00 & 130,79 & 134,72 & 134,72 & 134,72 \\
\hline M & 133,19 & 133,19 & 137,20 & 137,20 & 137,20 \\
\hline J & 133,63 & 134,44 & 138,46 & 138,46 & 138,46 \\
\hline J & 131,75 & 131,13 & 135,07 & 135,07 & 135,07 \\
\hline A & 135,12 & 137,43 & 141,53 & 141,53 & 141,53 \\
\hline S & 133,51 & 132,40 & 136,36 & 136,36 & 136,36 \\
\hline D & 133,79 & 134,28 & 138,29 & 138,29 & 138,29 \\
\hline N & 135,13 & 136,35 & 140,39 & 140,39 & 140,39 \\
\hline$D$ & 134,92 & 134,79 & 138,78 & 138,78 & 138,78 \\
\hline
\end{tabular}

La mejor alternativa es usar el factor de crecimiento dado por el ajuste de la línea de tendencia polinomial grado 3 ; es decir, usando el ajuste con mayor $\mathrm{R}^{2}$.

Tabla 20. Errores elevados al cuadrado

\begin{tabular}{|c|c|c|c|c|}
\hline \multirow{2}{*}{ Período } & Sin variación & $\begin{array}{c}\text { Última } \\
\text { variación }\end{array}$ & $\begin{array}{c}\text { Variaciones } \\
\text { promedio }\end{array}$ & ${\text { Mayor } \mathbf{R}^{\mathbf{2}}}$ \\
\cline { 2 - 5 } & $\begin{array}{c}\text { Torres con 0\% } \\
\text { Crecimiento }\end{array}$ & $\begin{array}{c}\text { Torres con 2,26\% } \\
\text { Crecimiento }\end{array}$ & $\begin{array}{c}\text { Torres con 2,296\% } \\
\text { Crecimiento }\end{array}$ & $\begin{array}{c}\text { Torres con 2,3\% } \\
\text { Crecimiento }\end{array}$ \\
\hline E & 5,76 & 29,16 & 40,96 & 40,96 \\
\hline F & 100,11 & 200,90 & 200,90 & 200,90 \\
\hline M & 31,59 & 93,26 & 93,26 & 93,26 \\
\hline A & 4,78 & 37,47 & 37,47 & 37,47 \\
\hline M & 21,07 & 73,89 & 73,89 & 73,89 \\
\hline J & 34,14 & 97,14 & 97,14 & 97,14 \\
\hline J & 6,40 & 41,89 & 41,89 & 41,89 \\
\hline A & 77,93 & 167,18 & 167,18 & 167,18 \\
\hline S & 14,47 & 60,15 & 60,15 & 60,15 \\
\hline D & 32,27 & 93,88 & 93,88 & 93,88 \\
\hline N & 60,10 & 139,06 & 139,06 & 139,06 \\
\hline D & 38,32 & 103,71 & 103,71 & 103,71 \\
\hline Totales & $\mathbf{4 2 6 , 9 2}$ & $\mathbf{1 1 3 7 , 7 0}$ & $\mathbf{1 1 4 9 , 5 0}$ & $\mathbf{1 1 4 9 , 5 0}$ \\
\hline
\end{tabular}

En este caso, también se puede men- determinar el valor de \%P. El mejor resulcionar que el resultado de los pronósticos tado se logró usando el método de Torres del método de Torres, no se ve mejorado, con $0 \%$ como valor del $\% \mathrm{P}$. cuando se usa el método del mayor $\mathrm{R}^{2}$ para 


\subsection{PRUEBA TRES}

Para realizar una tercera prueba se eligió una nueva serie de datos, que representan el consumo promedio de barriles ${ }^{25}$ de gaso- lina en Colombia en los últimos seis años, es decir una serie de 72 datos; a continuación pueden verse los datos originales:

Tabla 21.

\begin{tabular}{|l|c|c|c|c|c|c||}
\hline Meses & Año1 & Año2 & Año3 & Año4 & Año5 & Año6 \\
\hline ENERO & 4.051 .669 & 3.428 .972 & 3.465 .862 & 2.919 .208 & 2.835 .900 & 2.926 .130 \\
\hline FEBRERO & 3.583 .328 & 3.523 .296 & 3.120 .432 & 2.820 .748 & 2.540 .126 & 2.631 .980 \\
\hline MARZO & 3.957 .212 & 3.916 .447 & 3.411 .364 & 2.831 .137 & 2.733 .109 & 2.751 .183 \\
\hline ABRIL & 4.041 .930 & 3.493 .440 & 2.960 .790 & 2.773 .560 & 2.615 .014 & 2.505 .301 \\
\hline MAYO & 3.923 .608 & 3.666 .091 & 2.995 .530 & 2.846 .079 & 2.701 .131 & 2.704 .137 \\
\hline JUNIO & 3.783 .510 & 2.925 .570 & 3.151 .680 & 2.783 .370 & 2.602 .845 & 2.633 .125 \\
\hline JULIO & 4.068 .037 & 3.457 .771 & 2.836 .531 & 2.765 .355 & 2.754 .818 & 2.616 .546 \\
\hline AGOSTO & 3.909 .565 & 3.657 .721 & 3.299 .919 & 2.860 .773 & 2.849 .311 & 2.679 .817 \\
\hline SEPTIEMBRE & 3.882 .690 & 3.443 .310 & 2.987 .790 & 2.652 .210 & 2.693 .766 & 2.549 .386 \\
\hline OCTUBRE & 4.045 .035 & 3.339 .444 & 3.047 .796 & 2.779 .336 & 2.854 .967 & 2.680 .441 \\
\hline NOVIEMBRE & 3.616 .980 & 3.646 .680 & 2.847 .810 & 2.770 .260 & 2.766 .433 & 2.601 .652 \\
\hline DICIEMBRE & 4.387 .182 & 3.536 .945 & 3.360 .307 & 2.868 .585 & 3.070 .043 & 3.063 .701 \\
\hline
\end{tabular}

En la siguiente gráfica se puede observar el año 1998 hasta el año 2003, la información el comportamiento del consumo nacional de real del año 2004, se reserva para medir la gasolina (medido en barriles al mes), desde eficiencia del método de Torres.

Gráfica 8.

Consumo nacional de combustibles

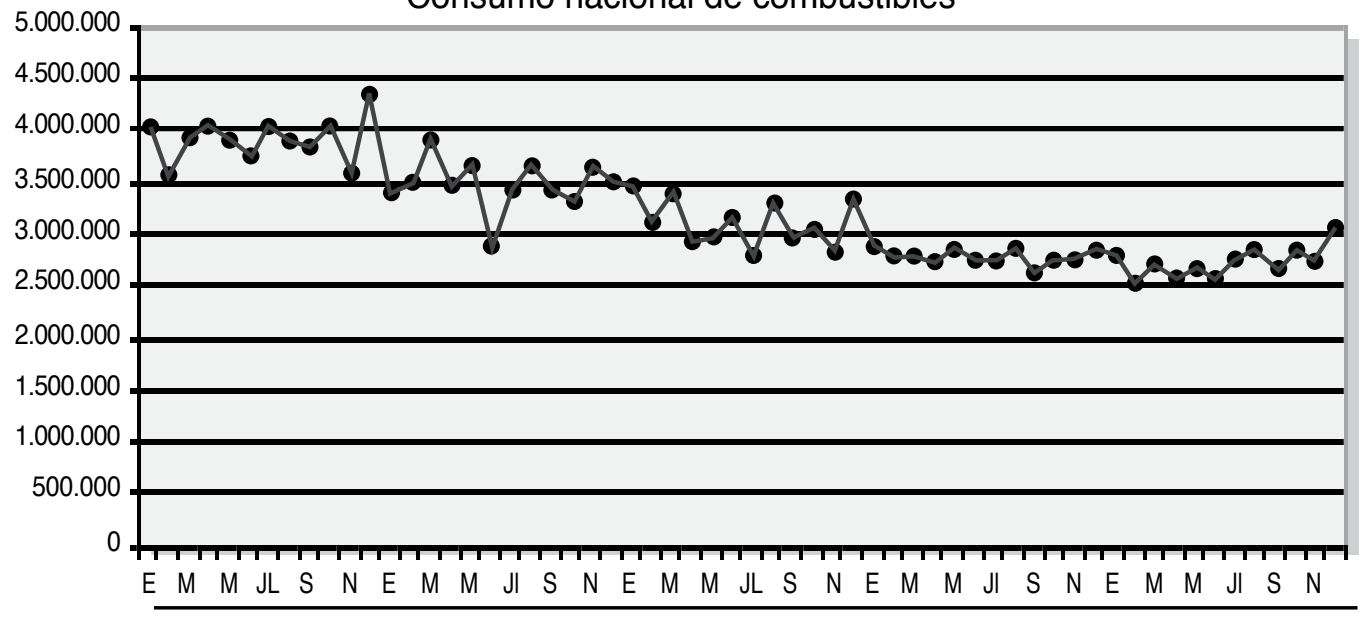

25. Consumo promedio de barriles de gasolina por mes, Fuente: Departamento Nacional de Planeación (DNP), puede accederse a esta serie de datos desde la página web: http://www.dnp.gov.co, en el apartado de Estadísticas 
El siguiente es el análisis del factor de crecimiento esperado de la serie, junto con las variaciones para cada período:

Tabla 22.

\begin{tabular}{|l|r|c|}
\hline \multicolumn{2}{|c|}{ Promedios } & $\begin{array}{c}\text { Cambio en el } \\
\text { promedio }\end{array}$ \\
\hline 1998 & $3.937 .562,17$ & \\
\hline 1999 & $3.502 .973,92$ & $-11,04 \%$ \\
\hline 2000 & $3.123 .817,58$ & $-10,82 \%$ \\
\hline 2001 & $2.805 .885,08$ & $-10,18 \%$ \\
\hline 2002 & $2.751 .455,22$ & $-1,94 \%$ \\
\hline Promedio & $3.224 .338,79$ & $-8,50 \%$ \\
\hline
\end{tabular}

A continuación puede verse el análisis completo de las series de crecimientos extrapoladas con las diferentes líneas de tendencia:

Gráficas 9.

Tendencia Lineal

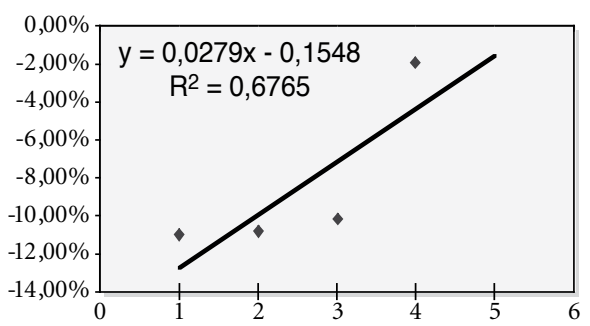

Tendencia Logarítmica

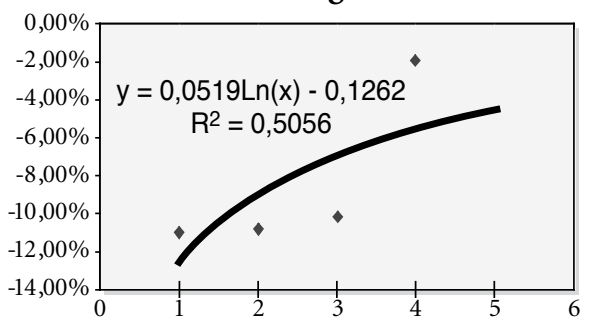

Polinomial 2

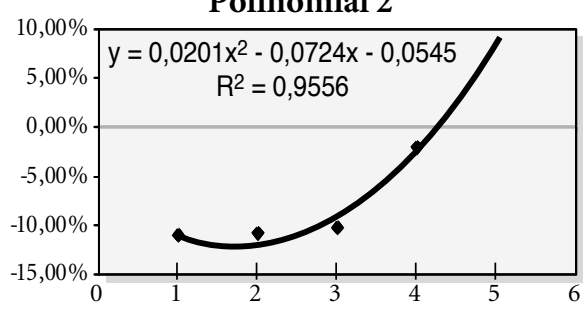

Polinomial 3

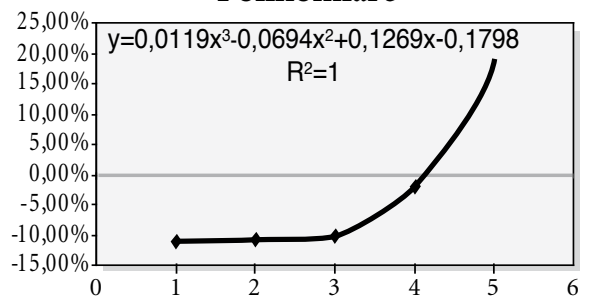

A continuación se resumen los resultados del análisis de tendencia de las variaciones de cada uno de los períodos y los valores esperados:

Tabla 23.

\begin{tabular}{|l|c|c|}
\hline Tendencias & $\mathbf{R}^{\mathbf{2}}$ & Variación \\
\hline Lineal & 0,6760 & $-2,0 \%$ \\
\hline Exponencial & $\mathrm{NA}$ & $0,0 \%$ \\
\hline Logarítmica & 0,5056 & $-3,6 \%$ \\
\hline Potencial & $\mathrm{NA}$ & $0,0 \%$ \\
\hline Polinomial 2 & $\mathbf{0 , 9 5 5 6}$ & $\mathbf{8 , 0} \%$ \\
\hline Polinomial 3 & $\mathbf{1 , 0 0 0 0}$ & $\mathbf{2 0 , 0} \%$ \\
\hline
\end{tabular}

Usando el Método de Torres para pronosticar las demandas de los siguientes 12 períodos, se tomaron como base histórica los últimos 12 meses, es decir el año más reciente, con diferentes factores de crecimiento \% $\mathrm{P}$, los resultados son: 
Tabla 24.

\begin{tabular}{|c|l|c|c|c|c|}
\hline \multirow{2}{*}{ Período } & Demanda & Sin variación & $\begin{array}{c}\text { Última } \\
\text { variación }\end{array}$ & $\begin{array}{c}\text { Variaciones } \\
\text { promedio }\end{array}$ & ${\text { Mayor } \mathbf{R}^{\mathbf{2}}}^{\text {real }}$ \\
\cline { 3 - 6 } & $\begin{array}{c}\text { Torres con 0\% } \\
\text { Crecimiento }\end{array}$ & $\begin{array}{c}\text { Torres con } \\
-1,94 \% \\
\text { Crecimiento }\end{array}$ & $\begin{array}{c}\text { Torres con } \\
-8,49 \% \\
\text { Crecimiento }\end{array}$ & $\begin{array}{c}\text { Torres con } \\
20 \% \\
\text { Crecimiento }\end{array}$ \\
\hline E & $2.926 .129,76$ & 2838652,00 & 2783583,00 & 2597651,00 & 3406383,00 \\
\hline F & $2.631 .979,81$ & 2452023,91 & 2404455,19 & 2243847,68 & 2942429,24 \\
\hline M & $2.751 .182,50$ & 2892366,90 & 2836255,73 & 2646805,25 & 3470840,91 \\
\hline A & $2.505 .301,24$ & 2491402,79 & 2443070,35 & 2279883,25 & 2989683,35 \\
\hline M & $2.704 .137,07$ & 2914986,65 & 2858436,50 & 2667505,29 & 3497984,63 \\
\hline J & $2.633 .124,90$ & 2569408,19 & 2519562,47 & 2351266,22 & 3083290,41 \\
\hline J & $2.616 .546,33$ & 2600738,59 & 2550284,27 & 2379936,60 & 3120886,90 \\
\hline A & $2.679 .816,57$ & 2732878,02 & 2679860,27 & 2500857,02 & 3279453,62 \\
\hline S & $2.549 .385,57$ & 2496049,75 & 2447627,14 & 2284135,65 & 2995260,27 \\
\hline D & $2.680 .441,45$ & 2822310,33 & 2767558,32 & 2582696,81 & 3386772,82 \\
\hline N & $2.601 .652,02$ & 2536239,55 & 2487036,63 & 2320913,56 & 3043487,84 \\
\hline D & $3.063 .700,93$ & 3134661,80 & 3073849,45 & 2868529,30 & 3761595,10 \\
\hline
\end{tabular}

Tabla 25. Errores elevados al cuadrado

\begin{tabular}{|c|c|c|c|c|}
\hline \multirow{2}{*}{ Período } & Sin variación & $\begin{array}{c}\text { Última } \\
\text { variación }\end{array}$ & $\begin{array}{c}\text { Variaciones } \\
\text { promedio }\end{array}$ & Mayor $\mathbf{R}^{2}$ \\
\hline & $\begin{array}{l}\text { Torres con 0\% } \\
\text { Crecimiento }\end{array}$ & $\begin{array}{l}\text { Torres con }-1,94 \% \\
\text { Crecimiento }\end{array}$ & $\begin{array}{l}\text { Torres con }-8,49 \% \\
\text { Crecimiento }\end{array}$ & $\begin{array}{l}\text { Torres con } 20 \% \\
\text { Crecimiento }\end{array}$ \\
\hline$E$ & $7.652 .358 .494,62$ & 20.319.578.786,50 & 107.898.295.771,14 & $230.643 .174 .530,50$ \\
\hline $\mathrm{F}$ & 224.776.353.917,44 & 272.144.355.376,26 & $465.508 .836 .564,50$ & $265.672 .904,55$ \\
\hline M & $1.139 .930 .736,80$ & 8.077.342.011,43 & 78.022.180.016,67 & $296.710 .233 .441,06$ \\
\hline$A$ & $188.987 .534 .576,67$ & 233.346.391.223,51 & $417.634 .550 .572,96$ & $4.039 .059 .226,36$ \\
\hline$M$ & $124.168 .928,44$ & $4.582 .376 .995,41$ & $66.886 .618 .344,48$ & $327.017 .987 .897,83$ \\
\hline J & $127.250 .277 .094,60$ & $165.296 .961 .014,97$ & $330.468 .095 .056,53$ & 24.699.471.358,39 \\
\hline$J$ & $105.879 .414 .392,91$ & $141.259 .832 .527,80$ & 298.326.966.699,10 & $37.930 .344 .594,34$ \\
\hline A & $37.346 .236 .479,29$ & $60.648 .660 .820,43$ & $180.856 .903 .098,09$ & $124.837 .749 .654,94$ \\
\hline$S$ & $184.968 .812 .284,76$ & $228.964 .758 .921,06$ & $412.156 .440 .614,25$ & $4.779 .028 .047,10$ \\
\hline 0 & $10.778 .473 .816,08$ & $25.144 .901 .766,43$ & $117.946 .189 .122,34$ & 212.192.026.781,13 \\
\hline $\mathrm{N}$ & $152.014 .378 .586,23$ & $192.802 .779 .766,17$ & $366.286 .647 .033,23$ & $13.772 .919 .888,94$ \\
\hline D & $43.485 .612 .073,73$ & $21.821 .106 .334,79$ & $3.317 .812 .853,76$ & $698.002 .339 .579,77$ \\
\hline Totales & $1.084 .403 .551 .381,56$ & $1.374 .409 .045 .544,75$ & $2.845 .309 .535 .747,04$ & $1.974 .890 .007 .904,91$ \\
\hline
\end{tabular}

En este caso, a diferencia de las series de del mayor $\mathrm{R}^{2}$, para este caso en particular, datos anteriores, el método de Torres no se la mejor alternativa es usar las $0 \%$ como ve mejorado por la técnica de escogencia \% 


\subsection{PRUEBACUATRO}

Para realizar una cuarta prueba se eligió una nueva serie de datos, que representan el consumo promedio de papel $^{26}$ en Colombia, dado en miles de toneladas de papel para el consumo interno, desde el año 1974 hasta el año 2003, es decir una serie de 30 datos. A continuación pueden verse los datos originales:

Tabla 26.

\begin{tabular}{|c|c|c|c|}
\hline Año & Producción & Año & Producción \\
\hline 1974 & 289 & 1989 & 511 \\
\hline 1975 & 248 & 1990 & 534 \\
\hline 1976 & 287 & 1991 & 563 \\
\hline 1977 & 295 & 1992 & 627 \\
\hline 1978 & 321 & 1993 & 610 \\
\hline 1979 & 342 & 1994 & 673 \\
\hline 1980 & 367 & 1995 & 677 \\
\hline 1981 & 372 & 1996 & 691 \\
\hline
\end{tabular}

\begin{tabular}{|c|c|c|c|}
\hline Año & Producción & Año & Producción \\
\hline 1982 & 366 & 1997 & 705 \\
\hline 1983 & 365 & 1998 & 711 \\
\hline 1984 & 412 & 1999 & 734 \\
\hline 1985 & 446 & 2000 & 771 \\
\hline 1986 & 457 & 2001 & 772 \\
\hline 1987 & 488 & 2002 & 848 \\
\hline 1988 & 504 & 2003 & 865 \\
\hline
\end{tabular}

En la siguiente gráfica (Producción de papel), se puede observar el comportamiento del consumo nacional de papel (medido en miles de toneladas al año), desde el año 1974 hasta el año 2003, se tomarán las demandas de los años 1994 hasta el año 1998 para pronosticar las demandas de los años 1999 hasta el año 2003, la información real de estos años se reservará para medir la eficiencia del método de Torres.

Gráfica 10.

\section{Producción de papel}

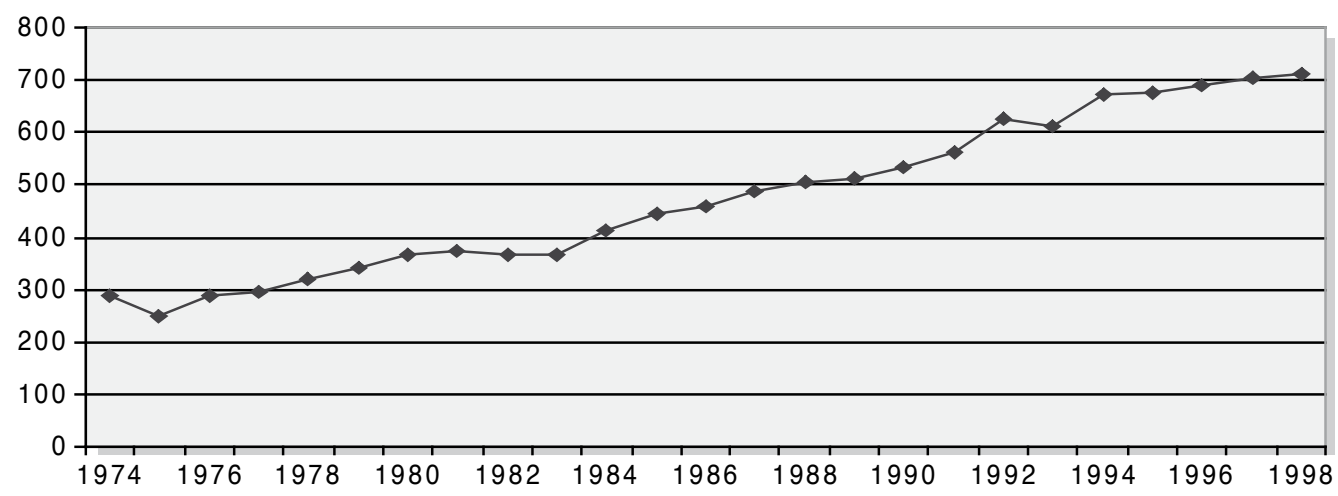

26. Consumo anual de papel en Colombia, desde el año 1974 hasta el año 2003, Fuente: Cámara de Pulpa, Papel y Cartón de la ANDI. Tomado de: Departamento Nacional de Planeación (DNP), puede accederse a esta serie de datos desde la página web: http://www.dnp.gov.co, en el apartado de Estadísticas 
En este caso en particular, no se tienen períodos de meses por año, sino que, se tienen los datos consolidados de cada año. Para calcular el factor de crecimiento o disminución esperado de la serie de datos en este caso se agrupó por grupos de cinco años y hacer la verificación de crecimientos o disminuciones entre períodos de cinco años. De este modo, las variaciones entre períodos quedan de la siguiente manera:

Tabla 27.

\begin{tabular}{|c|c|c|}
\hline Año & $\begin{array}{c}\text { Papel consumo } \\
\text { anual en miles } \\
\text { de toneladas }\end{array}$ & Variación \\
\hline 1974 & 289 & 0 \\
\hline 1975 & 248 & $-14,28 \%$ \\
\hline 1976 & 287 & $15,89 \%$ \\
\hline 1977 & 295 & $2,61 \%$ \\
\hline 1978 & 321 & $8,82 \%$ \\
\hline 1979 & 342 & $6,48 \%$ \\
\hline 1980 & 367 & $7,29 \%$ \\
\hline 1981 & 372 & $1,39 \%$ \\
\hline 1982 & 366 & $-1,48 \%$ \\
\hline 1983 & 365 & $-0,33 \%$ \\
\hline 1984 & 412 & $12,96 \%$ \\
\hline 1985 & 446 & $8,20 \%$ \\
\hline 1986 & 457 & $2,44 \%$ \\
\hline 1987 & 488 & $6,74 \%$ \\
\hline 1988 & 504 & $3,22 \%$ \\
\hline 1989 & 511 & $1,45 \%$ \\
\hline 1990 & 534 & $4,48 \%$ \\
\hline 1991 & 563 & $5,53 \%$ \\
\hline 1992 & 627 & $11,36 \%$ \\
\hline 1993 & 610 & $-2,77 \%$ \\
\hline 1994 & 673 & $10,30 \%$ \\
\hline 1995 & 677 & $0,64 \%$ \\
\hline 1996 & 691 & $2,01 \%$ \\
\hline 1997 & 705 & $2,09 \%$ \\
\hline 1998 & 711 & $0,87 \%$ \\
\hline Promedios & 474 & $3.84 \%$ \\
\hline
\end{tabular}

A continuación puede verse el análisis completo de las series de crecimientos extrapoladas con las diferentes líneas de tendencia:

\section{Gráficas 11.}

Tendencia Lineal

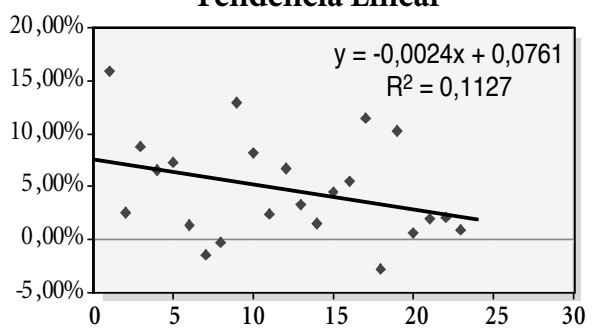

Tendencia Exponencial
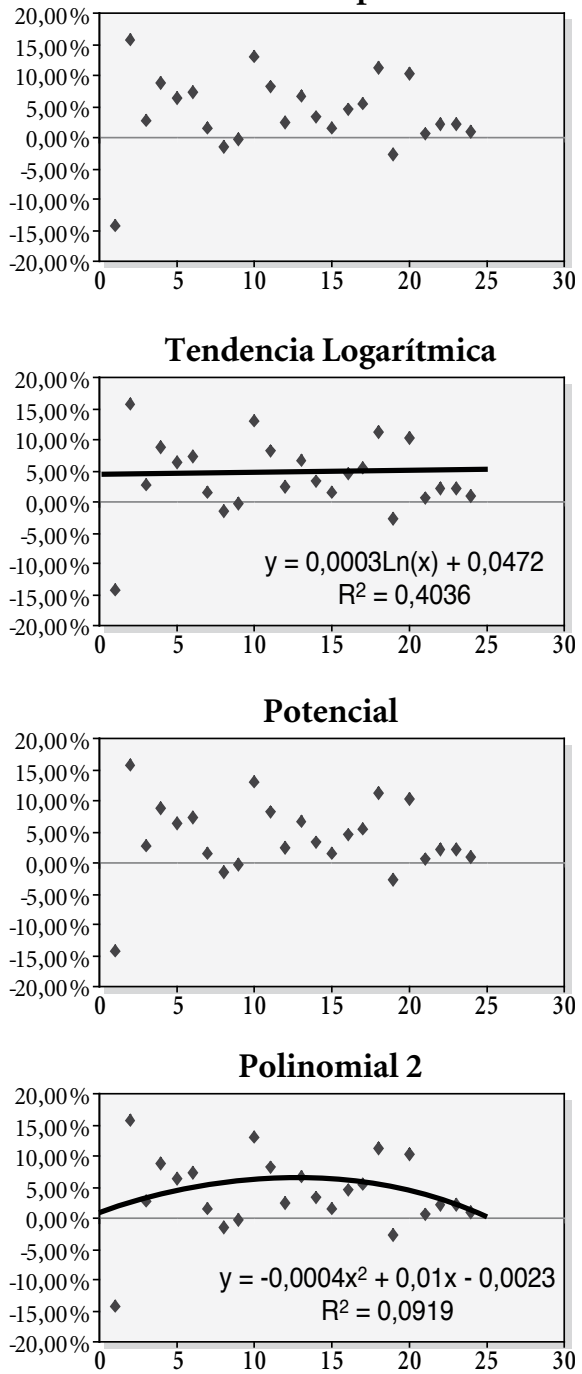


\section{Polinomial 3}

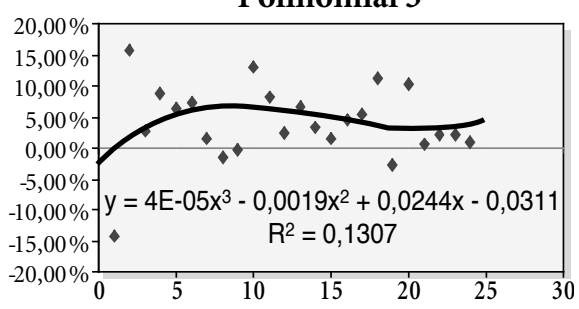

\section{Polinomial 4}

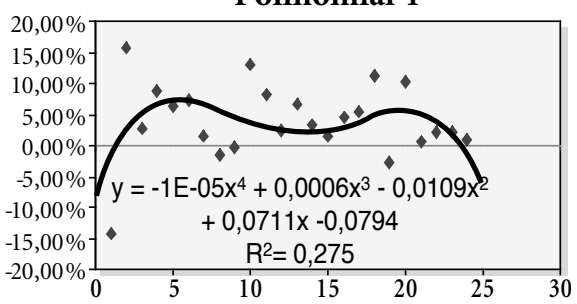

Polinomial 5

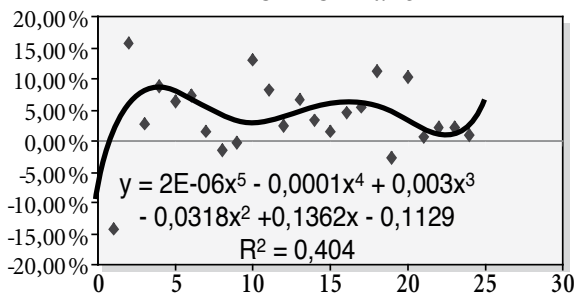

A continuación se resumen los resulta-

Usando el Método de Torres y usando dos del análisis de tendencia de las variacio- $\quad$ los diferentes factores de crecimiento \% nes de cada uno de los períodos y los valores los resultados son:

esperados:

Tabla 28.

\begin{tabular}{|c|c|c|}
\hline Tendencias & $\mathbf{R}^{\wedge \mathbf{2}}$ & Variación \\
\hline Lineal & 0,1050 & $2,0 \%$ \\
\hline Exponencial & NA & $0,0 \%$ \\
\hline Logarítmica & 0,0186 & $4,0 \%$ \\
\hline Potencial & NA & $0,0 \%$ \\
\hline Polinomial 2 & 0,6061 & $0,0 \%$ \\
\hline Polinomial 3 & 0,0719 & $2,0 \%$ \\
\hline Polinomial 4 & 0,0154 & $-6,0 \%$ \\
\hline Polinomial 5 & 0,2601 & $7,0 \%$ \\
\hline
\end{tabular}

Tabla 29.

\begin{tabular}{|l|c|c|c|c|c|}
\hline \multirow{2}{*}{ Período } & \multirow{2}{*}{$\begin{array}{c}\text { Demanda } \\
\text { real }\end{array}$} & \begin{tabular}{c} 
Sin variación \\
\cline { 3 - 6 }
\end{tabular} & $\begin{array}{c}\text { Última } \\
\text { Variación }\end{array}$ & $\begin{array}{c}\text { Variaciones } \\
\text { promedio }\end{array}$ & Mayor $\mathbf{R}^{\mathbf{2}}$ \\
\hline 1999 & 734 & 678 & $\begin{array}{c}\text { Torres con 0.87\% } \\
\text { crecimiento }\end{array}$ & $\begin{array}{c}\text { Torres con 4\% } \\
\text { crecimiento }\end{array}$ & $\begin{array}{c}\text { Torres } \\
\text { con 0\% } \\
\text { crecimiento }\end{array}$ \\
\hline 2000 & 771 & 684 & 684 & 706 & 678 \\
\hline 2001 & 772 & 692 & 698 & 712 & 684 \\
\hline 2002 & 848 & 710 & 717 & 721 & 692 \\
\hline 2003 & 865 & 715 & 722 & 749 & 710 \\
\hline
\end{tabular}


En la siguiente tabla se pueden ver los resultados de los errores elevados al cuadrado con respecto a los datos reales

Tabla 30.

\begin{tabular}{|c|c|c|c|c|c|}
\hline \multirow{2}{*}{ Período } & \multirow{2}{*}{$\begin{array}{c}\text { Demanda } \\
\text { real }\end{array}$} & Sin variación & $\begin{array}{c}\text { Última } \\
\text { variación }\end{array}$ & $\begin{array}{c}\text { Variaciones } \\
\text { promedio }\end{array}$ & Mayor $\mathbf{R}^{\mathbf{2}}$ \\
\cline { 3 - 6 } & & $\begin{array}{c}\text { Torres con 0\% } \\
\text { crecimiento }\end{array}$ & $\begin{array}{c}\text { Torres con 0.87\% } \\
\text { crecimiento }\end{array}$ & $\begin{array}{c}\text { Torres con 4\% } \\
\text { crecimiento }\end{array}$ & $\begin{array}{c}\text { Torres con 0\% } \\
\text { crecimiento }\end{array}$ \\
\hline 1999 & 734 & 29,16 & 129,96 & $1.115,56$ & 29,16 \\
\hline 2000 & 771 & 129,96 & 302,76 & $1.552,36$ & 129,96 \\
\hline 2001 & 772 & 376,36 & 645,16 & $2.342,56$ & 376,36 \\
\hline 2002 & 848 & $1.398,76$ & $1.971,36$ & $4.408,96$ & $1.398,76$ \\
\hline 2003 & 865 & $1.797,76$ & $2.440,36$ & $5.097,96$ & $1.797,76$ \\
\hline \hline Sumas & $\mathbf{3 . 9 9 0}$ & $\mathbf{3 . 7 3 2 , 0 0}$ & $\mathbf{5 . 4 8 9 , 6 0}$ & $\mathbf{1 4 . 5 1 7 , 4 0}$ & $\mathbf{3 . 7 3 2 , 0 0}$ \\
\hline
\end{tabular}

Para esta serie en particular, el método de Torres sí se ve mejorado gracias a la técnica de escogencia de la serie extrapolada usando el mayor $\mathrm{R}^{2}$. Para este caso, la mejor alternativa es usar el ajuste de la tendencia polinomial grado 2 . En este caso, el método de Torres por sí solo es igual de bueno al resultado del mayor $\mathrm{R}^{2}$.

\section{CONCLUSIONES}

7.1. El documento aporta una explicación más detallada del método de Torres y sirve para ampliar el modo adecuado de usar esta herramienta de pronóstico. Trata de mostrar las bondades y debilidades del método de Torres de acuerdo con su uso. Las bondades encontradas se pueden resumir en:

- En las pruebas, el método siempre ofrece mejores resultados con los datos más recientes, por lo cual, se sugiere al usar este método, trabajar con los datos más recientes del período que englobe la estacionalidad de la variable. Por ende, se puede concluir que basados en estas pruebas, no se requie- ren grandes colecciones de datos para pronosticar; esa es una fortaleza frente a otros métodos de pronóstico.

- El método de Torres involucra el \%P, el factor de crecimiento o decrecimiento de la serie para períodos futuros, ampliamente analizado a lo largo del documento. A pesar de ser un factor subjetivo, es el único método de pronóstico que contempla este factor.

- Las fortalezas encontradas en el apartado de análisis del método, muestran el buen desempeño que tiene el método frente a los comportamientos de ciclo y estacionalidad.

7.1. Las debilidades encontradas en el método de Torres son:

- El método siempre trata de replicar el comportamiento de los datos tomados como históricos, y en una serie con tendencia marcada en donde los primeros datos difieren mucho de los datos finales; el método en su pronóstico se parecerá mucho a los datos iniciales y no tiene en cuenta que la serie sigue creciendo o disminuyendo. 
- Otra debilidad encontrada se refiere al mismo factor $\% \mathrm{P}$, porque es una bondad cuando se puede predecir, de alguna manera, ese factor, pero cuando no se es un experto para predecir este factor, el método difiere mucho de los datos reales, y es muy complicado inferir el porcentaje exacto de aumento o disminución. Este trabajo pretende aportar un mecanismo que permitiera obtener, de una manera más objetiva, este factor de crecimiento de la serie.

7.3. De acuerdo con las pruebas realizadas al método con cuatro diferentes series, se puede ver que:

- En dos de las cuatro series, es decir, en el $50 \%$ de los casos, el método de Torres fue mejorado usando el \%P aportado por la extrapolación de las series de crecimiento o disminución usando la tendencia con mayor $\mathrm{R}^{2}$. No se puede generalizar este aspecto por el reducido número de pruebas, en cambio se propone hacer un número mayor de pruebas para comprobar si este porcentaje se mantiene o mejora.

- En los otros dos casos, es decir, en el $50 \%$ restante de las pruebas, el mejor resultado de precisión del método, se logró sin hacer variaciones y usando el resultado de Torres con $0 \%$ como valor de \% P. La recomendación es que se deban realizar más pruebas para comprobar que este comportamiento se mantenga..

- En ninguno de los casos, es decir el $0 \%$, se logró mejorar la precisión del método usando las últimas variaciones o las variaciones promedio.

- Se puede concluir que, el método de Torres por sí solo es mejor en el 50\% de los casos, frente al dato del \% $\mathrm{P}$ aportado por el análisis de los crecimientos de las series, por lo cual, a través de este documento se recomienda analizar la serie de datos y realizar la prueba frente a datos históricos reales, como se mostró en cada una de las cuatro pruebas. De este modo se puede mejorar el desempeño del método de Torres.

7.4. A través del estudio del método de Torres se aporta este documento que pretende un mejor entendimiento del método y ayudar en la forma de uso, tareas que no se presentan con claridad en el texto del cual se extrajo este método.

7.5. Se formula en hoja de cálculo el método de Torres para usarlo como herramienta de pronóstico, para cualquier número de observaciones históricas, que se desee (número de períodos), cosa que no se había hecho y que es de mucha utilidad para usarlo adecuadamente.

7.6. La medición pendiente es frente a otros métodos de pronóstico, para ello en un documento posterior se enfrentará el método de Torres con otros métodos de pronóstico para saber qué tan confiable es, y en dónde queda ubicado en el escalafón, con respecto a la confiabilidad de sus resultados. 


\section{BIBLIOGRAFÍA}

- FOGARTY, Donald (1999). Administración de la producción e inventarios. México: Editorial CECSA.

- HILLIER, Frederick (2001). Investigación de operaciones. Séptima edición. México: Editorial Mc Graw Hill.

- MAKRIDAKIS, Spyros (1997). Métodos de Pronóstico. México: Editorial Limusa.

- TORRES ACOSTA, Jairo Humberto (s.f.). Elementos de Producción. Bogotá: Editorial Universidad Católica de Colombia.

- PAREDES, Jorge (1994). Planificación de la Producción. México: Editorial Mc Graw Hill.

- PAREDES, Jorge. Planificación de la Producción. Universidad de Cuenca, Ecuador. En: http://rai.ucuenca.edu.ec/publicaciones/docentes/jorgeparedes.htm. Visitado en septiembre 3 de 2005.

- PAREDES, Jorge. Planificación de la Producción. El libro completo se puede bajar de: http://bibliotecavirtual.clacso.org.ar/ar/libros/ecuador/idiuc/teoria.doc

- PRAWDA, Juan (1999). Métodos y modelos de investigación de operaciones, métodos estocásticos. Vol. 2. Undécima edición. México: Editorial Limusa.

- TUROFF, Murray y LINSTONE, Harold (2002). The Delphi Method, Techniques and Applications. Massachussets: Addison Wesley Longman Publishing Co.

El libro completo en versión digital se puede consultar en: http://www.is.njit.edu/ pubs/delphibook/index.html. Visitado en Octubre 11 de 2005.

- VÉLEZ, Pareja Ignacio (2004). Decisiones empresariales bajo incertidumbre. Bogotá: Editorial Norma.

- Página Web del Departamento Nacional de Planeación, apartado de Estadísticas. Visitado en julio 12 de 2005. En: www.dnp.gov.co 